Wright State University

CORE Scholar

9-1989

\title{
Micronuclear Genome Organization in Euplotes crassus: a Transposonlike Element Is Removed during Macronuclear Development
}

\author{
Scott Everet Baird \\ Wright State University - Main Campus, scott.baird@wright.edu \\ Gina M. Fino \\ Susan L. Tausta \\ Lawrence A. Klobutcher
}

Follow this and additional works at: https://corescholar.libraries. wright.edu/biology

Part of the Biology Commons, Genetics Commons, Medical Sciences Commons, and the Systems Biology Commons

\section{Repository Citation}

Baird, S. E., Fino, G. M., Tausta, S. L., \& Klobutcher, L. A. (1989). Micronuclear Genome Organization in Euplotes crassus: a Transposonlike Element Is Removed during Macronuclear Development. Molecular and Cellular Biology, 9 (9), 3793-3807.

https://corescholar.libraries.wright.edu/biology/160

This Article is brought to you for free and open access by the Biological Sciences at CORE Scholar. It has been accepted for inclusion in Biological Sciences Faculty Publications by an authorized administrator of CORE Scholar. For more information, please contact library-corescholar@wright.edu. 


\title{
Micronuclear Genome Organization in Euplotes crassus: a Transposonlike Element Is Removed during Macronuclear Development
}

\author{
SCOTT E. BAIRD, † GINA M. FINO, SUSAN L. TAUSTA, AND LAWRENCE A. KLOBUTCHER* \\ Department of Biochemistry, University of Connecticut Health Center, Farmington, Connecticut 06032
}

Received 13 April 1989/Accepted 18 June 1989

\begin{abstract}
After mating, hypotrichous ciliated protozoa transform a set of their micronuclear chromosomes into thousands of short, linear DNA molecules that form the macronuclear genome. To examine micronuclear genome organization in the hypotrich Euplotes crassus, we have analyzed two cloned segments of micronuclear DNA as well as the macronuclear DNA molecules that are derived from them. $E$. crassus was found to display a number of features characteristic of other hypotrich genomes, including (i) clustering and close spacing of the precursors of macronuclear DNA molecules, (ii) the frequent occurrence of internal eliminated sequences within macronuclear precursors, (iii) overlapping macronuclear precursors, (iv) lack of telomeric repeats at the ends of macronuclear precursors, and ( $v)$ alternative processing of the micronuclear chromosome to yield multiple macronuclear DNA molecules. In addition, a moderately repetitive, transposonlike element that interrupts the precursors of two macronuclear DNA molecules has been identified and characterized. This transposonlike element, designated Tec1, is shown to be reproducibly removed from one of the macronuclear precursors during independent episodes of macronuclear development.
\end{abstract}

Hypotrichous ciliated protozoa undergo an extensive genome reorganization process during their life cycles (for reviews, see references 32 and 47 ). The ability of these unicellular organisms to drastically alter their genome yet maintain genetic continuity stems from the fact that each cell contains two distinct nuclei. The micronucleus, which contains chromosome-sized DNA, is transcriptionally inactive but plays a major role during the sexual phase of the life cycle and is often referred to as the germ line nucleus. In contrast, the macronucleus is responsible for all nuclear transcription during vegetative growth and is equivalent to a somatic nucleus. The macronuclear genome is unusual in that it is composed of 10,000 to 20,000 different short, linear, gene-sized DNA molecules with an average size of approximately 2 kilobase pairs $(\mathrm{kbp})$. There are generally 1,000 or more copies of each linear DNA molecule per macronucleus, and each appears to be an independent genetic unit which contains the information required to encode and express a single gene product $(20,27)$ as well as to allow its own replication.

Despite its unusual genetic organization, the macronucleus replicates and divides during each vegetative cell cycle. However, after the sexual phase of the life cycle, the macronucleus is destroyed and a new one is generated from a mitotic copy of the micronucleus. It is during this process of macronuclear development that extensive genomic changes occur, giving rise to the unique genetic organization of the macronucleus. At the cytological level, macronuclear development begins with the replication of the micronuclear chromosomes to form polytene chromosomes. The polytene chromosomes are then broken into many pieces, and the individual fragments are encased in vesicles. Once the vesicles form, extensive but selective DNA degradation

\footnotetext{
* Corresponding author.

† Current address: Department of Molecular Genetics, Albert Einstein College of Medicine, 1300 Morris Park Avenue, Bronx. N.Y. 10461.
}

occurs, resulting in the loss of up to $95 \%$ of the sequence complexity of the micronuclear genome. The vesicles then break down, and the remaining DNA molecules undergo additional rounds of replication to form the highly amplified macronuclear genome. Thus, macronuclear development includes steps of micronuclear chromosome fragmentation, DNA elimination, and DNA amplification.

On the basis of the comparison of cloned macronuclear DNA molecules with their developmental precursors, a number of additional features of micronuclear genome organization and reorganization have been deduced. First, macronuclear DNA molecules have been found to be clustered in the micronuclear genome and are generally separated by fewer than 500 bp $(9,34,25,26)$. Second, macronuclear telomeres, consisting of repeats of the octanucleotide $5^{\prime}$ CCCCAAAA-3' $\left(\mathrm{C}_{4} \mathrm{~A}_{4}\right.$ repeats), are not present at the ends of the micronuclear precursors of macronuclear DNA molecules but are added during the course of macronuclear development $(11,31,39)$. Third, the micronuclear precursors of macronuclear DNA molecules frequently contain short blocks of DNA (internal eliminated sequences [IESs]) that are not present in the mature macronuclear DNA molecule $(23,31,40)$, which implies that nucleic acid splicing also occurs during development. In Oxytricha nova, IESs range in size from 14 to $500 \mathrm{bp}$, and approximately 60,000 have been estimated to occur in the micronuclear genome (40).

Large, moderately repetitive DNA elements that are eliminated during development also exist in the micronuclear genome, but their significance is poorly understood $(9,25$, 26). These elements are often found in association with clusters of macronuclear DNA precursors, and Jahn et al. (25) have presented preliminary evidence that such a repetitive element interrupts the micronuclear precursor of a macronuclear DNA molecule in Euplotes crassus. Similarly, Herrick et al. (22) have found that two members of a transposonlike family of elements interrupt a putative precursor of a macronuclear DNA molecule in Oxytricha fallax. 
These results suggest that development removal of transposons may occur during macronuclear development.

The above general features of macronuclear development are not unique to the hypotrichous ciliates. Holotrichous ciliates, such as Paramecium tetraurelia and Tetrahymena thermophila, undergo similar types of developmental DNA rearrangement events $(15,28)$, although to a more limited extent. For example, macronuclear development in $T$. thermophila entails only a few hundred chromosome fragmentation events (3) and approximately 5,000 DNA breakage and joining events similar to those of the IES removal phenomenon described above (50).

Although the above studies have provided insights into the type of events occurring during hypotrich macronuclear development, they provide little information on the molecular mechanisms involved in the various rearrangement events. Further progress in this area would be aided greatly by an experimental system that would allow one to follow the processing of defined segments of micronuclear DNA during the course of macronuclear development and ultimately to develop in vitro systems for particular rearrangement processes. Unfortunately, the most well-studied hypotrichs at the molecular level present major difficulties in pursuing such studies. Stylonychia lemnae has a very complex micronuclear genome (5) with a variable chromosome constitution (4). This has made it difficult to obtain cloned precursors of macronuclear DNA molecules. In contrast, well-characterized cloned segments of micronuclear DNA are available for the hypotrichs $O$. nova and $O$. fallax, but the common laboratory strains of these organisms have lost the ability to mate efficiently and to produce viable sexual offspring.

In view of these limitations, we have sought to develop the hypotrich $E$. crassus as a model organism to study the dynamics of macronuclear development. E. crassus has a relatively small micronuclear genome of $1.7 \times 10^{9} \mathrm{bp}(32)$, so that obtaining recombinant clones of desired regions of the micronuclear genome is relatively straightforward. In addition, this organism possesses a defined mating system (19) that allows control of the sexual cycle and, hence, of macronuclear development. The ability to generate large numbers of cells synchronously proceeding through macronuclear development and the utility of the system for detecting developmental DNA intermediates have both been demonstrated $(42,43)$.

In this report, we address the question of whether genome organization and macronuclear development in E. crassus are similar to those in previously studied hypotrichs. Two cloned segments of micronuclear DNA containing the precursors of macronuclear DNA molecules have been isolated and characterized in detail. The results indicate that the organizations of the $E$. crassus genomes is generally very similar to those of previously studied hypotrichs. In contrast to other previously studied species, however, two macronuclear gene precursors were found to be interrupted by large, repetitive, transposonlike elements. Additional studies demonstrate that the repetitive element is reproducibly removed from macronuclear gene precursors during development.

\section{MATERIALS AND METHODS}

$E$. crassus cell lines. The $E$. crassus cell lines used in these studies were derived from cells originally isolated from a site in San Terenzio, Italy by P. Luporini (University of Camerino, Italy). Recombinant DNA libraries were constructed from strain G1, a partially inbred mating type I cell line $\left(\mathrm{mt}^{1} / \mathrm{mt}^{1}\right.$; see reference 19 for a discussion of the mating type system). Other cell lines used were $\mathrm{NcC10}$, a mating type III cell line $\left(\mathrm{mt}^{3} / \mathrm{mt}^{1}\right)$, and strains CC102, CC103, CC104, and $\mathrm{CC} 106$, which were derived from a mating of $\mathrm{G} 1$ and $\mathrm{NcC} 10$.

$E$. crassus cell lines were grown and maintained in supplemented artificial seawater (Instant Ocean; Aquarium Systems, Mentor, Ohio) with the marine alga Dunaliella salina as a food source as described by Roth et al. (42).

DNA isolation. For the isolation of total cellular DNA, 10 to 20 liters of $E$. crassus were grown until the algal food supply was exhausted and then were starved for an additional 1 to 2 days. The cultures were then filtered through a 35- $\mu \mathrm{m}$ nitex membrane (Tetko, Inc., Lancaster, N.Y.) to remove large algal debris. The cells were harvested by collection on a $15-\mu \mathrm{m}$ nitex membrane, and the concentrated cells were centrifuged at $100 \times g$ for $1 \mathrm{~min}$. DNA was then isolated from cells as described for the hypotrichous ciliate O. nova (33).

Micronuclear DNA was isolated from total cellular DNA by taking advantage of the fact that micronuclear DNA has a high molecular weight, while the macronuclear DNA consists of small molecules. Thus, it was possible to purify micronuclear DNA from macronuclear DNA by centrifugation of whole-cell DNA through linear 5 to $20 \%$ potassium acetate gradients (36). Alternatively, total cellular DNA was subjected to electrophoresis in a $1 \%$ low-melting-point agarose gel, and the region of the gel containing high-molecularweight DNA was excised. The gel slice was then melted by heating it to $65^{\circ} \mathrm{C}$ and was loaded on a second $1 \%$ lowmelting-point agarose gel. The micronuclear DNA was again excised, digested in situ with the appropriate restriction enzyme when required, and purified from the gel as described by Kuhn et al. (35).

DNA from recombinant bacteriophage was purified as described by Maniatis et al. (36). Recombinant plasmid DNA was prepared as described by Godson and Vapnek (17) with minor modifications (36).

Construction and screening of recombinant libraries. The construction of the E. crassus $\mathrm{G} 1$ macronuclear library (LEMAC library) has been described previously (6). In brief, synthetic EcoRI linkers were added to total macronuclear DNA that had been previously treated with EcoRI methylase and blunt ended by treatment with T4 DNA polymerase. The resulting DNA molecules were then ligated into the EcoRI site of the bacteriophage vector $\lambda \mathrm{gt} 10$ (24). The resulting recombinant clones contain essentially full-length macronuclear DNA molecules and lack only the single-stranded telomeric region (33).

An $E$. crassus $\mathrm{G} 1$ micronuclear recombinant DNA library (LEMIC library) was constructed by first performing a series of partial Sau3A digestions on total micronuclear DNA, pooling the digests, and fractionating them by electrophoresis on a $1 \%$ low-melting-point agarose gel. Sau3A fragments in the 9.4 to $23 \mathrm{kbp}$ size range were excised from the gel, eluted, and ligated with lambda EMBL3 vector arms (16) (Stratagene, Inc., San Diego, Calif.) that had been generated by digestion with BamHI. The resulting DNA was packaged into phage particles by using the Gigapack system (Stratagene, Inc.). A total of $5.5 \times 10^{5}$ recombinant phage were produced and amplified on plates before storage of the library. On the basis of a micronuclear genome size of $1.7 \times$ $10^{9} \mathrm{bp}$ (32), the library was estimated to have a 0.96 probability of containing any micronuclear sequence.

The recombinant libraries were screened by using the plaque lift method of Benton and Davis (8).

Restriction endonuclease digestions, gel electrophoresis, and 
Southern hybridization. DNAs were digested with restriction enzymes in accordance with the directions of the manufacturer (New England BioLabs, Inc., Beverly, Mass., and Bethesda Research Laboratories, Bethesda, Md.). Sizes of DNAs were determined by electrophoresis through agarose or low-melting-point agarose gels prepared with and run in TBE ( $89 \mathrm{mM}$ Tris borate, $89 \mathrm{mM}$ boric acid, $2 \mathrm{mM}$ EDTA).

For hybridization analyses, DNA was transferred to nitrocellulose filters (46), which were then incubated with radiolabeled probes at $65^{\circ} \mathrm{C}$ as previously described (34). Unless otherwise noted, the filter received two 30 -min washes in $2 \times$ $\mathrm{SSC}-0.5 \%$ sodium dodecyl sulfate (SDS) $(1 \times \mathrm{SSC}$ is $0.15 \mathrm{M}$ $\mathrm{NaCl}$ plus $0.015 \mathrm{M}$ sodium citrate) and then two 30-min washes in $0.1 \times \mathrm{SSC}-0.5 \%$ SDS.

Restriction fragments used as hybridization probes were excised directly from low-melting-point agarose gels and radio labeled with $\left[\alpha-{ }^{32} \mathrm{P}\right] \mathrm{dATP}$ by the random hexamer priming method (13).

For quantitative analysis of Southern blots, band intensities on autoradiograms were compared by using a model 1650 scanning densitometer (Bio-Rad Laboratories, Rockville Centre, N.Y.) interfaced with an Apple II plus computer (Apple Computer, Inc., Cupertino, Calif.) equipped with Chromatochart software (Interactive Microware, Inc., State College, Pa.). Alternatively, two-dimensional scanning densitometry was performed by using a Bio-Rad model 620 video densitometer and 2-D Analyst software (version 1.10).

DNA sequence analysis. DNA fragments were cloned into bacteriophage M13 mp10 or M13 mp11 and sequenced by the dideoxy-chain termination method (44), using kits purchased from New England BioLabs or United States Biochemical Corp. (Cleveland, Ohio) and $\left[\alpha-\right.$ thio- ${ }^{35}$ S $]$ dATP as the labeled nucleotide triphosphate. In some cases the chemical cleavage sequencing method was used with DNA fragments labeled at a $3^{\prime}$ end (37).

\section{RESULTS}

Isolation and characterization of $\boldsymbol{E}$. crassus micronuclear recombinant clones. Two recombinant clones containing segments of E. crassus micronuclear DNA were isolated from the LEMIC bacteriophage lambda library, and the developmental fates of sequences in the clones were determined. The first clone, LEMICD, which contained a 13.2$\mathrm{kbp}$ segment of micronuclear DNA, was selected from a group of randomly chosen micronuclear clones on the basis of its ability to hybridize to macronuclear DNA. The second clone, LEMICV, contained a 14.2-kbp micronuclear DNA insert and was isolated during a screen of the micronuclear library by using a previously cloned $0.9-\mathrm{kbp}$ macronuclear DNA molecule referred to as V2 (clone pMACV2) as a hybridization probe. Restriction maps of these two micronuclear clones were generated and are shown in Fig. 1.

To determine the developmental fates of sequences within each of these clones, a series of restriction fragments spanning the length of the clones was generated, and each fragment was used individually to probe a Southern blot of native (i.e., undigested) E. crassus total cellular DNA. Conceivably, these clones could contain three types of sequences which were distinguishable in the hybridization analysis: (i) sequences which give rise to macronuclear DNA molecules during development: (ii) unique, or low-copynumber, micronuclear DNA sequences that are eliminated during the development of the macronucleus; and (iii) highly repetitive micronuclear DNA sequences that are eliminated during macronuclear development. In the Southern hybrid- ization analyses, regions of the micronuclear clones that give rise to macronuclear genes will show hybridization to discrete. low-molecular-weight DNA molecules $(<20 \mathrm{kbp})$. In contrast, the micronuclear DNA contained within the total cellular DNA has a high molecular weight and migrates at the limit of mobility in the agarose gels. Under the conditions used, unique and low-copy-number micronuclear-limited sequences showed no hybridization, as homologous sequences are present in very small amounts. Repetitive micronuclear-limited sequences ( $>100$ copies per genome), however, showed hybridization to high-molecular-weight, limited-mobility DNA. For these experiments, as well as for others presented below, it was assumed that high-molecularweight DNA is equivalent to micronuclear DNA. Micronuclear DNA has been demonstrated to be chromosomal and of high molecular weight in a number of hypotrich species (for a review, see reference 32 ), but it remains possible that some macronuclear sequences also fall into the high-molecularweight class.

The results of this type of analysis are shown for clone LEMICD (Fig. 2). Fragments A, B, C, and D, which were derived from the left end of the clone, hybridized exclusively to high-molecular-weight DNA and hence contained repetitive micronuclear DNA sequences that are eliminated during macronuclear development (Fig. 1A and 2). Similarly, the left end of clone LEMICV contained repetitive micronuclear-limited sequences (Fig. 1B).

Both clones also contained regions that hybridized to macronuclear DNA molecules. In the case of LEMICD, restriction fragments $E, F$, and $G$, derived from the central region of the cloned micronuclear DNA segment, detected 1.65- and/or 1.55-kbp macronuclear DNA molecules (Fig. 1A and 2), indicating that these regions of LEMICD contain the micronuclear precursors of these macronuclear DNA molecules. We have designated the 1.65 - and $1.55-\mathrm{kbp}$ macronuclear DNA molecules D7 and D8, respectively. The rightward region of LEMICV shared homology with a number of macronuclear DNA molecules (Fig. 1B). Fragments derived from this clone hybridized to 3.8-, 0.90- (this corresponds to the cloned V2 macronuclear DNA molecule used to select clone LEMICV), 1.8-, and 1.6-kbp macronuclear DNA molecules (V1, V2, V3, and V4, respectively, in Fig. 1). Fragments $\mathrm{L}$ and $\mathrm{M}$, located near the right end of LEMICV, hybridized to both V3 and V4 (Fig. 1B). This presumably represents a case of alternative processing in which two macronuclear DNA molecules are derived from the same segment of micronuclear DNA, as has been observed for other hypotrich species $(23,30)$.

The remaining regions of LEMICD (fragments $\mathrm{H}, \mathrm{I}, \mathrm{J}$, and K. Figs. 1A and 2) showed no hybridization to whole-cell DNA and thus contained unique or low-copy-number sequences that are eliminated during macronuclear development.

Isolation and characterization of macronuclear DNA molecules derived from LEMICD and LEMICV. Recombinant clones of all of the macronuclear DNA molecules derived from LEMICD and LEMICV were isolated to characterize their micronuclear organization more thoroughly. This was done by using selected restriction fragments from the two micronuclear clones to screen a recombinant library of macronuclear DNA molecules (the LEMAC library). The clones isolated were designated by the name of the macronuclear DNA molecule preceded by LEMAC (e.g., LEMACD7, Fig. 1A) or by $p$ in cases in which the macronuclear DNA insert was subcloned into the plasmid vector pUC12 (49) to facilitate analysis (e.g., pMACV3; Fig. 1B). 
A.

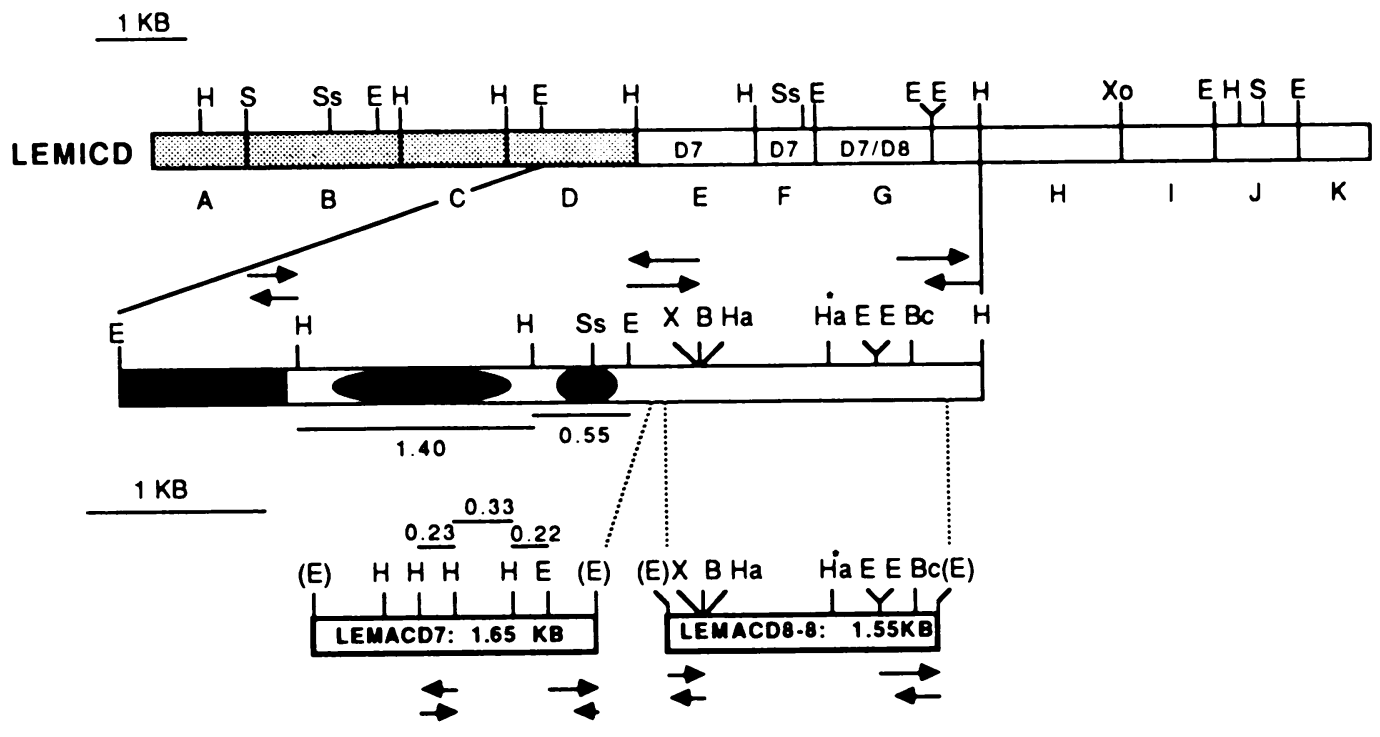

B.

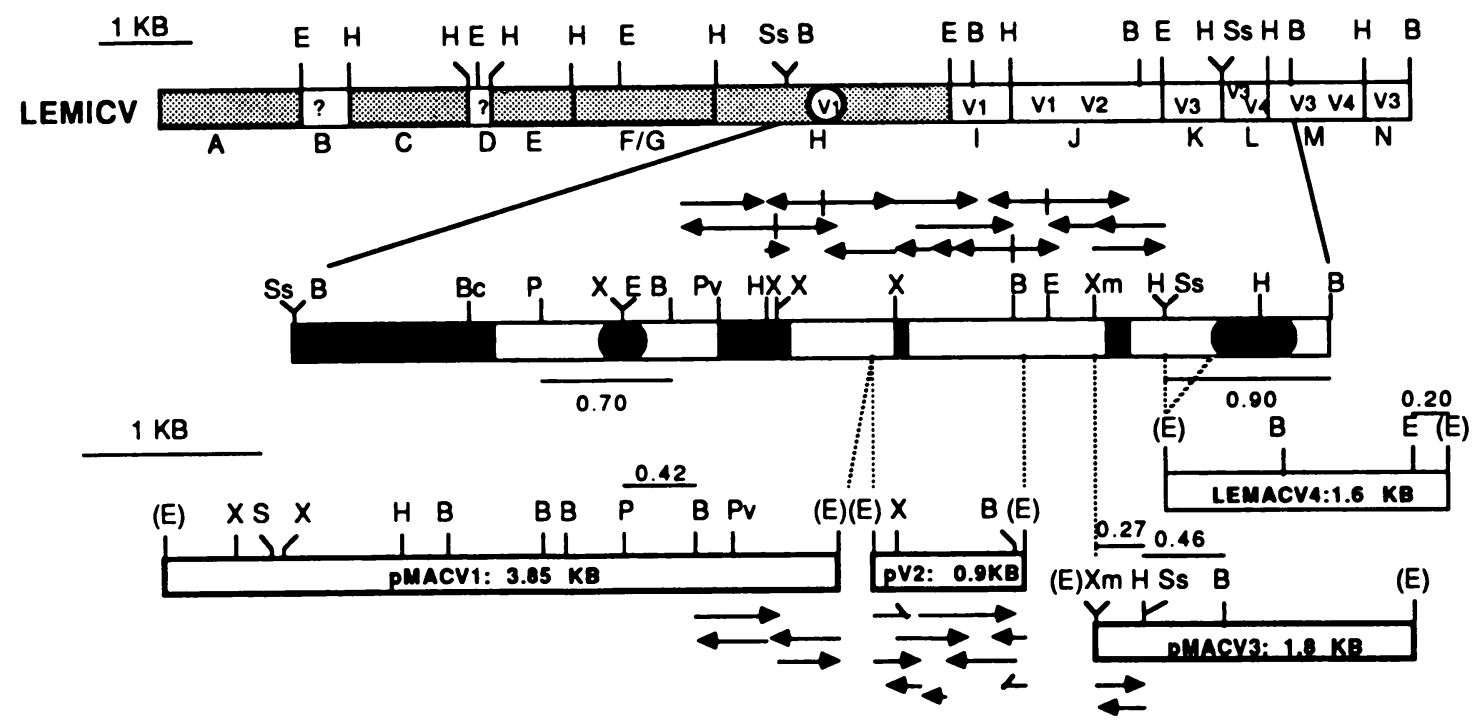

FIG. 1. Restriction maps of cloned micronuclear and macronuclear DNA segments. (A) Restriction map of LEMICD. Boxed areas of map and associated letters (A, B, C. etc.) indicate restriction fragments used in hybridization experiments. Regions of LEMICD containing micronuclear repetitive DNA ( $\square)$ and micronuclear-limited unique or low-copy-number sequences ( $\square$ ) are shown, and regions which hybridized with macronuclear DNA molecules contain the name of the homologous macronuclear gene (D7 or D8). The region of LEMICD containing the precursors of macronuclear genes is enlarged for comparison with the restriction maps of the macronuclear clones LEMACD7 and LEMACD8-8. Positions of macronuclear gene ends in the enlarged region of the micronuclear clone (…) are shown. IESs whose positions and sizes are precisely known $(\boldsymbol{\square})$ and IESs whose precise positions have not been determined $(\boldsymbol{O})$ are indicated. Regions sequenced by the dideoxy-chain termination method $(\rightarrow)$ and the chemical cleavage methods $(\rightarrow)$ are indicated. The sizes of selected restriction fragments referred to in the text are indicated. A variable Haelll site in the macronuclear D8 gene is indicated (*). Note that the terminal bracketed $E c o$ RI sites of the macronuclear clones were created during the cloning process and are not present at the ends of native macronuclear DNA molecules. H, HindIII: E. EcoRI: S. SalI: Ss. SstI: Xo. XhoI: X. XbaI: B, BglII: Ha. HaeIII; Bc, Bc/l: P, PstI; Pv, PvuII; and Xm, XmnI. Note that all Bc/I and BglII sites have not been mapped, and HaellI sites were only mapped in LEMACD8-8 and the corresponding region of LEMICD. (B) Restriction map of the micronuclear clone LEMICV. with the region containing macronuclear gene precursors enlarged. Also shown are restriction maps of the macronuclear gene clones pMACV1. pMACV2. pMACV3. and LEMACV4. Other aspects of the figure are as described for panel A. Note that not all Bc/l. Pv'ull. and Xmnl sites are mapped.

Restriction mapping and hybridization experiments utilizing the macronuclear clones localized the regions of the micronuclear clones responsible for giving rise to these macronuclear DNA molecules (Fig. 1; data not shown). Once this level of mapping was accomplished. DNA-se- quencing studies were undertaken to localize the macronuclear gene precursors more precisely and examine their organization in detail. In the case of LEMICD, regions spanning the right end of the D7 gene, and both ends of the D8 gene were sequenced along with the corresponding 


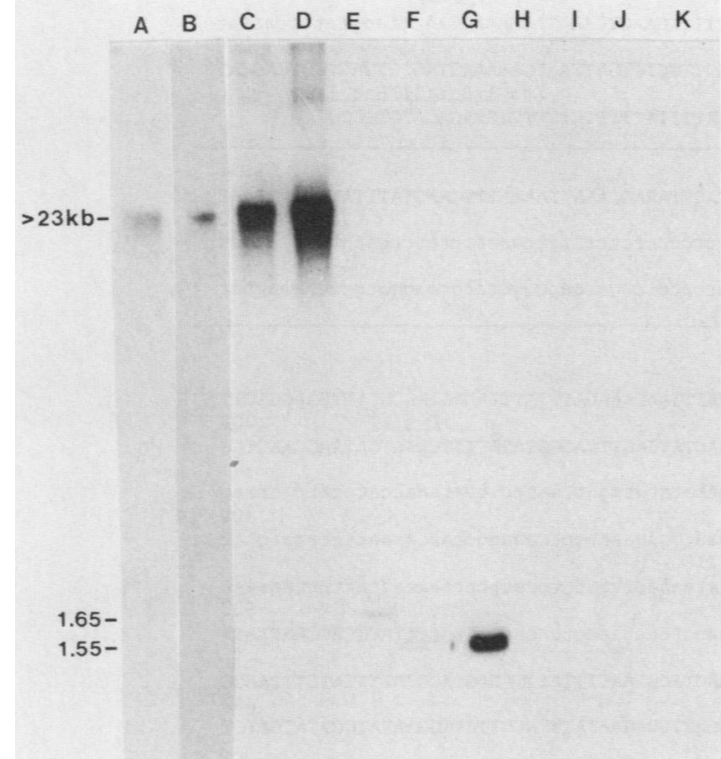

FIG. 2. Hybridizations of LEMICD restriction fragments to Southern blots containing undigested $E$. crassus whole-cell DNA. A Southern blot was prepared from a gel containing multiple lanes of $E$. crassus whole-cell DNA, and the blot was cut into strips. The blot strips were individually hybridized with restriction fragments $A$ through K of LEMICD (Fig. 1A), and the strips were reassembled before autoradiographic exposure (probe B was hybridized to an independent Southern blot). Sizes of hybridizing fragments are indicated on the left in kilobase pairs. Note that fragments $F$ and $G$ also showed weak hybridization to $1.65-\mathrm{kbp}$ DNA molecules upon longer autoradiographic exposure.

termini of the cloned macronuclear DNA molecules (Fig. 1A). The sequences of these regions are shown in Fig. 3A and $3 \mathrm{~B}$. The sequences of the corresponding regions of the macronuclear and micronuclear clones were in complete agreement, providing strong evidence that the micronuclear clones do indeed contain the true developmental precursors of these macronuclear DNA molecules. However, as has been observed for other hypotrichous ciliates $(11,31,39)$, the micronuclear precursors of the D7 and D8 macronuclear DNA molecules lacked the telomeric $C_{4} A_{4}$ repeat sequences present at the ends of all macronuclear DNA molecules. These repeats are added to macronuclear DNA molecules during the course of macronuclear development. The sequencing results also indicated that the micronuclear copies of D7 and D8 are in close proximity, with only a 36-bp intergenic spacer separating them (Fig. 3A). It should be noted that two variant forms of D8, defined by the presence or absence of a HaeIII restriction site (Fig. 1A), exist in the macronuclear genome of $E$. crassus $\mathrm{G} 1$, which was used to construct the recombinant libraries. In previous genetic analyses we have determined that these two forms are allelic (6). The micronuclear and macronuclear clones analyzed here both contain the HaeIII-plus form of D8.

In the case of LEMICV, a more extensive sequencing analysis was undertaken. A 2,740-bp segment of the micronuclear clone (extending from the $B g$ III site at the right end of the V1 gene precursor, through the entire region giving rise to V2, and up to the HindIII site at the left end of the V3 precursor) and the corresponding regions of the $\mathrm{V} 1, \mathrm{~V} 2$, and V3 macronuclear clones were sequenced (Fig. 1B). As described above, the sequences of the macronuclear and micronuclear clones were in complete agreement (Fig. 3C), with the exception of the macronuclear telomeric repeats and a number of IESs which are discussed further below. In the micronucleus, the right end of $\mathrm{V} 2$ is separated from the left end of $\mathrm{V} 3$ by a 400 -bp intergenic spacer. In contrast, the right end of V1 overlaps the left end of V2 by $6 \mathrm{bp}$; that is, V1 and V2 share 6 bp of micronuclear DNA.

The sequence analysis included the entire V2 macronuclear DNA molecule. V2 was found to be 814 bp in length, excluding the telomeric repeats. The function of V2 is not clear, as no unambiguous open reading frame was found within this macronuclear DNA molecule. The region most likely to be involved in coding for a protein is an open reading frame extending from base 1276 to base 1533 of the sequence as shown in Fig. $3 \mathrm{C}$. This region has a relatively high $\mathrm{G}+\mathrm{C}$ content of $49.7 \%$, which is a feature of coding regions in ciliated protozoa (28) and an elevated RNY/YNR ratio (2.27), which is a general characteristic of coding regions in many organisms (45). However, this open reading frame does not begin with an initiation codon, which may indicate that the protein coding region of $\mathrm{V} 2$ is interrupted by an intron. Analysis of the transcript produced by $\mathrm{V} 2$ will be required to clarify the function, if any, of this macronuclear DNA molecule.

Finally, as noted above, hybridization analyses suggested that V3 and V4 are derived from the same region of LEMICV by alternative processing (Fig. 1B). The sequencing data provide a strong indication that the $\mathrm{V} 3$ macronuclear DNA molecule is derived from the region of the micronuclear genome represented in clone LEMICV. No sequencing studies have been performed on the macronuclear V4 DNA molecule, so that alternative processing has not been rigorously demonstrated. However, cross-hybridization analyses performed with the two macronuclear DNA clones and LEMICV indicated that if alternative processing is involved it is likely to be complex, involving at least two distinct types of alternative rearrangement events. First, while the majority of V3 and V4 were found to crosshybridize, the 0.27-kbp HindIII-to-end fragment of V3 did not hybridize to V4, and the $0.20-\mathrm{kbp} E c o$ RI-to-end fragment of V4 did not hybridize to V3 (data not shown). Thus, the termini of V3 and V4 are distinct, and if a single region of micronuclear DNA gives rise to both of these macronuclear DNA molecules, then alternative use of chromosome fragmentation sites is likely involved. Second, there are a number of differences in the restriction maps of the two macronuclear DNA molecules and the cross-hybridizing region of LEMICV (Fig. 1B), suggesting that alternative internal rearrangements must also occur. For example, fragment N of LEMICV (Fig. 1B) hybridized to V3 but not to $\mathrm{V} 4$, indicating that this region would have to be removed from V4 but not from V3.

IESs in the micronuclear precursors of macronuclear DNA molecules. On the basis of the above sequencing analyses and additional hybridization analyses, a number of conventional IESs were defined in the regions of LEMICD and LEMICV that give rise to macronuclear DNA molecules during development (large regions of repetitive DNA also interrupt the V1 and D7 precursors and are discussed in the following section). Comparison of the sequenced region of LEMICV with the corresponding sequenced regions of the macronuclear clones revealed that IESs of 374,31 , and 144 bp interrupt the micronuclear copies of the V1, V2, and V3 genes, respectively (Fig. $3 \mathrm{C}$ ). In common with IESs sequenced in other hypotrichs $(23,31,40)$, each IES is bounded by a short direct repeat, one copy of which is retained in the mature macronuclear DNA molecule. The 
A.

D7R -

TGCAGTACAGCTTGATGTMUITCATCTATCTTTTTATTMUTATATTMCTAGTTCATTTTTCAACTCMUTACUUACGMtgt aggtattccattat ggct tatagtatttctcCAATTTIAGAGTACAGTAGCTGMTTMATMTTATTCCTGATTTTCTTTGATTMTGUUUITAGTCTGAGTCCTCAGCAT Xba_L/Bal II/Hae III ATTGGCMITTGCAGTCTGGGCTACMAGATCAAGAGCMATCTTCTCMTAGATTGTCTGATTTTATTTTTCTCTTCTCTAGAGATCTGGCC

B. D8RGTTTATTGTCTTCTATTTGAGGTTTGCACACTCCCTCCTGTACAMCGTTCATTTCCCTACTTMAGCAMATTAMATTTCAAGTATTTATMTTTTMC
200 tttcataaatattgaaaatattat tgattattaaataaaatgcatattgagggatcttatccccctctttttgtgaaatcctatctgaagtttctaaact atgctaaattctttgtaactaaactaatatt cgct tgt gtgt tgaaacct ggaagaaccacctccgaagaggggctatccaagggtgggaaaaat tat C. Boilis AGATCTTATAGAGCTAGAGTITTAMAMATTAAATTTTAGMCTAGGCTGCGTAGACATTTMTTAAGATTTATTTAGAGAAGCGTTGTGAAGGACTT

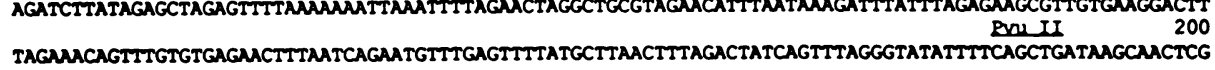

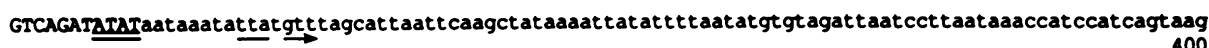
agtcaaataaatgtttaaataacagaatat ggcaaat $t t t$ caaagatt tggagt aaataaat ggagaaatcctcaaaggccaacaaaat tct aaat ctat Hind III
ggggtacataactttgagtatttaatagaacaaataatgggtaaagtctgraaagct ataaactgtttgtccattctcaaataacattct tagaaat
600 gagttattttgtctagaattttcatgttgttatgaataataaagt tctagattatttt aactcaataacgtaactatgtaaraf TACTCAGCAGTTATA AGGCTGGAGMACTCCTTCAAGAGTTCATTGGTTATGGTATGUATATGMATTTMACMCTACGTMATTTTATTTAGAGTACGTATTTTATGTTTMACC TATTTCACCGGMTTGAGATAGGTGAMGTTTTGGGTTATTTMMATTTGTMTGMAGGAGATTGCAGUTTATTTAGGTTCTTGGUITATCCATATGATCT GTTTGATAGTTGGACTTGGCTTAGUUAGAGGTTATGGATAGTGTGTAGAGGGTTATTCTTTCCGTCTCTMUGACTATCAGTTCACCAAGACCTMAC TCTTTTGATGGCTGATGTMAGCTCTTGTGGTATTTTTAGUTGMGGATTCATTTATAGGUATTMUATATGGTAGATTTTTTGTTTTMTTATGUUT TTATTTGAGATYTTGGGTGACTTACTCTAGTTCAACTCTTTTATGTTTCGTTCGCCTAGATTTTGTACCTCTTTTATAUCTCTTTCGCTCATATTTAM

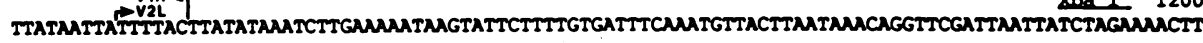

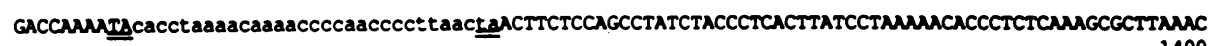

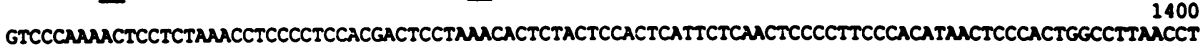
TCCTGAAAACCCTACTAATCThCCCGACCCCCTTAGTTCCTCCCTTTTCCCAGTTATCTCCAGTTTGGTTCTTACCCGATCGGGCMAGUAACTGAGTGG CTTTTGGGGTGTTTTGGGGTTTGGCCTGGTGATTAATGAGATGTGTATTAGGTGTTGTAATGATTTGTTTGATTGGGAGGGATTATTTTTMMGNuNA ATATAGGGTATGTMATATAATTGGAACAGTAATTTTGAGTTTATTTCAATTAGAAATAUAGGGGCTAGATATGATALATCTGTTGTCNAGNMAG

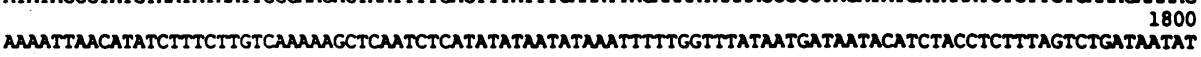
ATTTGCTATMATTCATCCCCTTATCAAAACTATAGTTAGCTCCTTAACTCTTMACTCTGTTTMATCTTATCTTCAMTTTMACCATAMTTATACCCCA
V2R
BQI II AAAGATCTAATACAATCACCATTTATAATCTAACCAACCCCCACCACTTAAATCCCCtaaattcccaacctcccct cacctctacccccaacccct CaA gtaatctcccaaccccctaatectcccctcttcacct ccaccctcgt tcctcccctcccacctaccccctattatct cagt cattctcccatccataaac Eco BI 2200 ttcttggctgaattctgagccgtggtatagtctgccagagcacgtgtgttt taggtgaggttgaggtagatggtttggggt taaggggaattatggggtg ggggagagggggttttgggggtgagattggtggaaggggt traagagggtttt gggtggaattgggt tatttggggggttaaatt tegtattttecgt
2400

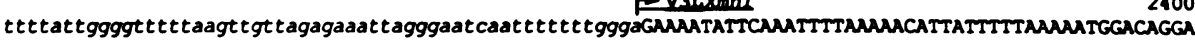
CTAAGACACAGAGGAAGAAGAGGAAGTAAGGCTatatt gcaaaat $t$ gataaagggtataagagaatagtagagactt tgagt cataaaaattagaata
2600 tctgaggcttctcatcgccatactaactcaacactcctagaataaaaatatcct tcttattcaatatcccatat tcataATACTGACTATATTITCTCTT TTATCCCTTATAATTCGTATATTTCTTCCTAATTATCAAATGGTATATTTCCAGTCTTTTGGACCTAITTACCCTCAITCAACAAATGAAACCAGATT TATTCCCTAGAACTATCTATACATACCATTTTGAAGC III

FIG. 3. Sequences of regions of micronuclear clones LEMICD and LEMICV. (A) Sequence of the region of clone LEMICD containing the right end of the precursor of the D7 macronuclear DNA molecule, a 36-bp intergenic spacer, and the left end of the D8 precursor. (B) Sequence of the region of clone LEMICD containing the right end of the D8 precursor and flanking micronuclear DNA. (C) Sequence of the region of clone LEMICV beginning at the BglII site at the right end of the V1 precursor, extending through the entire V2 precursor, and ending at the HindIII site at the left end of the V3 precursor. In each case, sequences which will form macronuclear DNA molecules are shown in uppercase letters, IESs are shown in lowercase letters, and intergenic spacers are shown in lowercase italic letters. The positions of the ends of macronuclear gene precursors are indicated (e.g., D7R is the right end of the D7 precursor). A number of restriction sites are shown to aid alignment with the restriction maps shown in Fig. 1. The direct repeats at the ends of IESs are doubly underlined, and IES inverted repeats are underlined by arrows, with gaps denoting mismatches. The sequences of macronuclear DNA molecules D7, D8, V1, V2, and V3 are not explicitly shown, but they were found to be identical to the LEMICD and LEMICV sequences shown, with the exception of the IESs indicated. In addition, all ends of the cloned macronuclear DNA molecules possessed $28 \mathrm{bp}$ of the telomeric repeat sequence $\mathrm{C}_{4} \mathrm{~A}_{4}$.

direct repeats for the 374-, 31-, and 144-bp IESs are 5'ATAT-3', 5'-TA-3', and 5'-ATA-3', respectively. Short imperfect inverted repeats, also a common feature of IESs in other hypotrichs, were found near the termini of the 374- and 144-bp IESs but not near the termini of the small 31-bp IES (Fig. 3C).
In addition to the IESs detected in the sequenced region of LEMICV, evidence for other IESs in the two micronuclear clones was obtained in hybridization analyses. For example, the 420-bp restriction fragment extending from the PstI site to the rightmost $B g$ III site of the macronuclear V1 gene hybridized to the 700-bp PstI-BglII fragment of LEMICV 


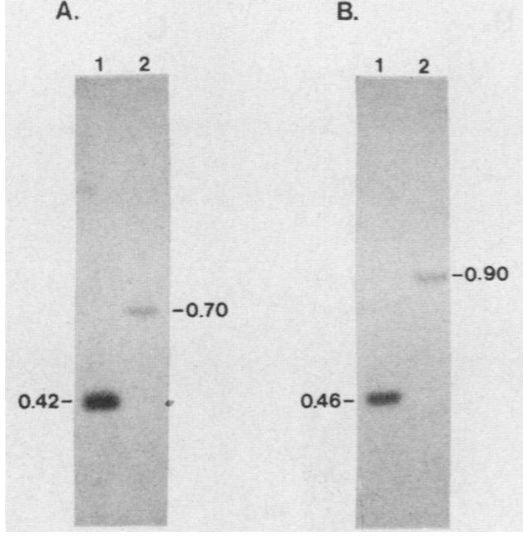

FIG. 4. Detection of IESs by hybridization analysis. (A) Hybridization of the 0.42-kbp PstI-BglII fragment of pMACV1 to a Southern blot containing PstI-BgllI digests of pMACV1 (lane 1) and LEMICV (lane 2). (B) Hybridization of the 0.46-kbp SstI-Bg/II fragment of pMACV3 to a Southern blot containing Sst I- and BgllI-digested pMACV3 (lane 1) and LEMICV (lane 2). Sizes of hybridizing fragments are indicated in kilobase pairs. For both $\mathrm{A}$ and $\mathrm{B}$, filters were washed at $65^{\circ} \mathrm{C}$ in $1 \times \mathrm{SSC}-0.5 \%$ SDS.

(Fig. 1B and 4A). This implies that one or more IESs totalling approximately $280 \mathrm{bp}$ are removed from this region during the maturation of the V1 gene. In the case of V3, the 460-bp SstI-BglII fragment of the macronuclear clone hybridized to a 900-bp SstI-BglII fragment in LEMICV (Fig. $1 \mathrm{~B}$ and $4 \mathrm{~B}$ ), indicating that one or more IESs totaling $440 \mathrm{bp}$ are present in this region of the V 3 precursor. Similarly, the 220-bp EcoRI-HindIII fragment near the right end of macronuclear D7 corresponded to a 550-bp EcoRI-HindIII fragment in micronuclear clone LEMICD, and the 330-bp HindIII fragment of LEMACD7 corresponded to a 1,400-bp HindIII fragment in LEMICD, implying that one or more IESs totalling 330 and $1,070 \mathrm{bp}$ are present in the respective regions of the micronuclear copy of D7 (Fig. 1A; data not shown).

The single macronuclear gene precursor for which no evidence of IESs exists is the D8 gene. No IESs were found within the regions of D8 sequenced (Fig. 3A and B), and all restriction fragments derived from internal regions of the micronuclear and macronuclear copies of D8 appeared to be identical in size when subjected to side-by-side gel electrophoresis. This is the first instance in which a micronuclear copy of a gene in a hypotrichous ciliate has been found to lack an IES.

Large repetitive DNA elements interrupt the precursors of V1 and D7. During the above analyses it became evident that the entire precursors of V1 and D7 were not contained in their respective micronuclear clones, despite the fact that the inserts were sufficiently large to contain them in their entirety. To determine the extent of V1 sequences in the micronuclear clone, LEMICV was hybridized to a Southern blot of EcoRI- and BglII-digested pMACV1 DNA. Only the 0.78- and 0.74-kbp fragments were detected (Fig. 5A); these fragments correspond to approximately the right half of macronuclear V1 (see Fig. 7). Similarly, LEMICD hybridized only to fragments to the right of and including the 0.33-kbp HindIII fragment of D7 (Fig. 1A; data not shown).

Comparisons of the macronuclear and micronuclear restriction maps indicated that the repetitive DNA sequences in both micronuclear clones were at or near the boundaries of the V1 and D7 homologous regions and thus appear to

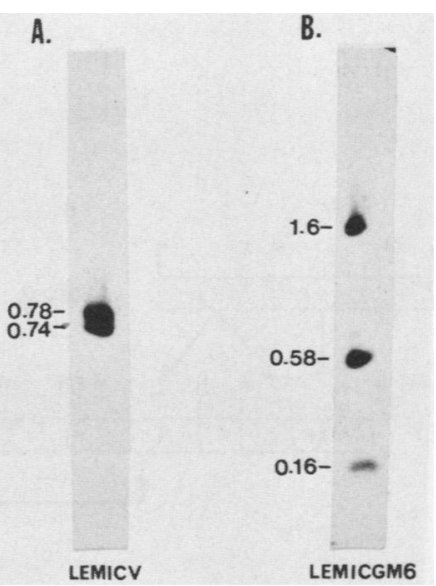

FIG. 5. Hybridizations of LEMICV (A) and LEMICGM6 (B) to Southern blots containing pMACV1 doubly digested with $B g l I I$ and EcoRI. Sizes of hybridizing fragments are indicated in kilobase pairs. Filters were washed in $1 \times \mathrm{SSC}-0.5 \%$ SDS at $65^{\circ} \mathrm{C}$.

interrupt the precursors of these macronuclear DNA molecules. As such, it was of interest to further characterize the blocks of repetitive DNA.

To determine whether the repeated DNA regions of the two clones were related, restriction fragments from the repetitive region of $\mathrm{LEMICD}$ were isolated and used to probe Southern blots containing restriction digests of LEMICV. The results of this analysis are summarized in Fig. 6A. Fragment C of LEMICD hybridized to fragment A of LEMICV, while fragment D of LEMICD hybridized to both fragments $A$ and C of LEMICV (note that under the gel electrophoresis conditions used, the small restriction fragments B and D of LEMICV ran off the gel and were not included in this analysis). Other restriction fragments of LEMICD and LEMICV showed no detectable hybridization to the opposite clone. Thus, the two clones contain related repetitive DNA elements. However, two lines of evidence indicate that the repetitive elements in the two clones are not identical. First, the restriction maps of the cross-hybridizing regions of the two clones cannot easily be aligned (Fig. 6A). Second, repetitive fragments $E, F / G$, and $H$ of LEMICV did not hybridize with LEMICD.

To determine whether the repetitive regions represented a large repetitive element or were composed of a smaller tandemly repeated unit, a series of intraclonal hybridizations were performed (data not shown). With the exception of fragments $\mathrm{C}$ and $\mathrm{H}$ of LEMICV (Fig. 6A), none of the restriction fragments derived from the repetitive regions of the micronuclear clones hybridized to other fragments within the clone, indicating that the repetitive regions represent a large element (the basis for the cross-hybridization of fragments $\mathrm{C}$ and $\mathrm{H}$ is discussed below). The entire repetitive region will be referred to as a Tec1 element (the basis for this designation is given in the Discussion).

To investigate the general organization of related Tec1 elements in the micronuclear genome, repetitive restriction fragments of LEMICD were used to probe Southern blots containing $E$. crassus whole-cell DNA digested with a number of restriction enzymes. These results are summarized in Table 1. Hybridizations with fragment C of LEMICD were representative of the overall results. Fragment C, a 1.1-kbp HindIII fragment, showed hybridization to discrete fragments in whole-cell DNA digested with a number of restriction enzymes as well as lesser hybridization to a broad 
A.

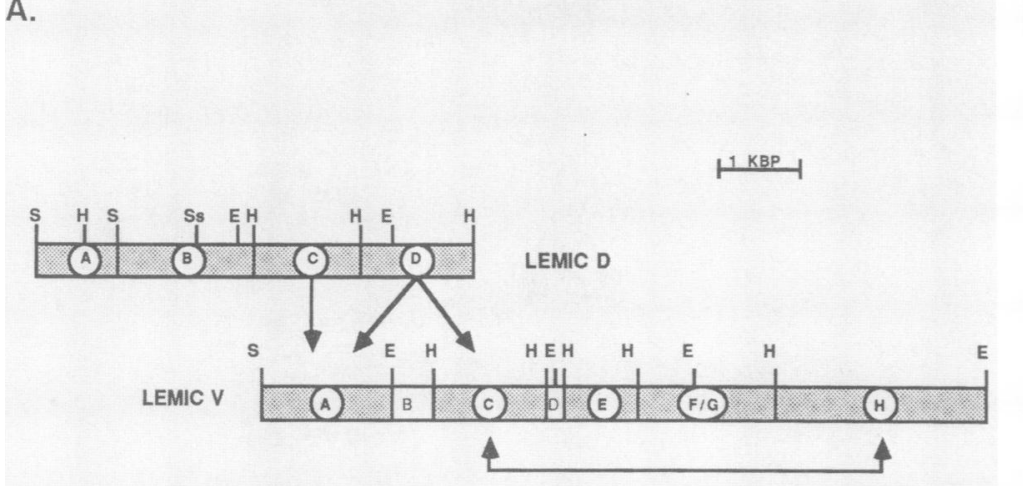

B.

B.
C. $\begin{array}{lllll}H & E & 3 & 4 & 5\end{array}$

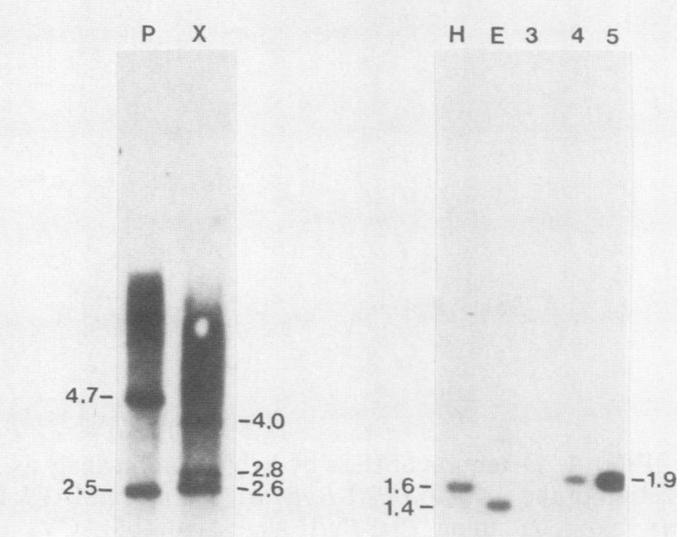

FIG. 6. Characterization of repetitive sequences in LEMICD and LEMICV. (A) Summary of cross-hybridization analyses for repetitive regions of LEMICD and LEMICV. Restriction maps of the repetitive regions of the two clones are shown. Letters denoting restriction fragment probes are as described for Fig. 1. Arrows indicate fragments of LEMICD that cross-hybridized with fragments of LEMICV. The maps have been aligned such that cross-hybridizing regions of the two clones overlap. Cross-hybridization between LEMICV fragments C and $\mathrm{H}$ is also indicated. (B) Autoradiographs from a Southern blot containing total cellular DNA digested with PstI (lane P) and $X b a \mathrm{I}$ (lane $\mathrm{X}$ ) and probed with the 1.1-kbp HindIII fragment of LEMICD (fragment C, Fig. 1A). Sizes of the major hybridizing fragments are indicated in kilobase pairs. (C) Hybridization of the 1.1-kbp HindIII fragment of LEMICD to a Southern blot containing HindIII (lane H) and EcoRI (lane E)-digested whole-cell DNA with various amounts of EcoRI-digested LEMICD DNA, calculated to be equivalent to $10^{3}, 10^{4}$, and $10^{5}$ copies per cell of the 1.1-kbp HindIII fragment (lanes 3, 4, and 5, respectively) relative to the amounts of cellular DNA present in the HindIII and EcoRI lanes. The LEMICD lanes were used as copy-number standards for scanning densitometry.

spectrum of DNA fragments as revealed by a general background smear (Fig. 6B and C). We interpret these results as indicating that most of the Tecl elements in the micronuclear genome have a conserved restriction map, but that degenerate or rearranged elements which have lost or gained restriction sites are also present. The elements in both LEMICD and LEMICV differed from the conserved element and thus appear to be degenerate copies. For example, fragment $\mathrm{C}$ of LEMICD detects predominantly $1.4-$ and $1.6-\mathrm{kbp}$ fragments in EcoRI and HindIII digests of whole-cell DNA (Fig. 6C), respectively, while the corresponding EcoRI and HindIII fragments in the clone are of 1.9 and $1.1 \mathrm{kbp}$, respectively (Fig. 1A).

These genomic hybridization experiments also provided a minimum estimate of the size of the majority of the Tec1 elements in the micronuclear genome. Fragments A, B, C, and D of LEMICD, which encompass a contiguous region of $5.4 \mathrm{kbp}$ in LEMICD, all detected 4.7-kbp PstI fragments in whole-cell DNA (Table 1). This suggests that sequences corresponding to the four LEMICD fragments are generally adjacent in other repetitive elements in the genome. The PstI sites that define the 4.7-kbp genomic fragments are appar-

TABLE 1. Summary of hybridization analyses on repetitive regions of micronuclear clones

\begin{tabular}{|c|c|c|c|c|c|}
\hline \multirow{2}{*}{ Hybridization probe } & \multicolumn{4}{|c|}{ Size (kbp) of homologous fragment in total cellular DNA digested with: } & \multirow{2}{*}{ Copies per cell ${ }^{a}$} \\
\hline & $\overline{\text { EcoRI }}$ & HindIII & $P s t \mathrm{I}$ & $X b a \mathbf{I}$ & \\
\hline \multicolumn{6}{|l|}{ LEMICD fragments } \\
\hline A $(1.0-\mathrm{kbp} S a l \mathrm{I})$ & 1.5 & 1.8 & $4.7,2.5$ & $4.0,2.6$ & $2.1 \times 10^{4}$ \\
\hline B (1.85-kbp Sall-HindIII) & 1.7 & Smr $r^{b}$ & $4.7,2.5,1.3$ & $4.0,2.8,2.6,1.25$ & $5.0 \times 10^{2}$ \\
\hline C (1.1-kbp HindIII) & 1.4 & 1.6 & $4.7,2.5$ & $4.0,2.8,2.6$ & $1.5 \times 10^{4}$ \\
\hline D (1.5-kbp HindIII) & 1.5 & 1.4 & $4.7,2.5$ & $4.0,2.8$ & $1.3 \times 10^{4}$ \\
\hline LEMICGM6 fragment (0.28-kbp BglII-AatII) & $\mathrm{Smr}$ & $1.9,1.4$ & $\mathrm{Smr}$ & Smr & $6.4 \times 10^{4 c}$ \\
\hline
\end{tabular}

" Copy numbers are based on densitometric comparisons of the predominant homologous fragments in total cellular DNA with known amounts of cloned DNA, as in Fig. 6C.

${ }^{b}$ Smr, No discrete fragments detected; only a general smear of hybridization was observed.

" Hybridization was done at $55^{\circ} \mathrm{C}$; filter was washed in $2 \times \mathrm{SSC}-0.5 \%$ SDS at $55^{\circ} \mathrm{C}$. 


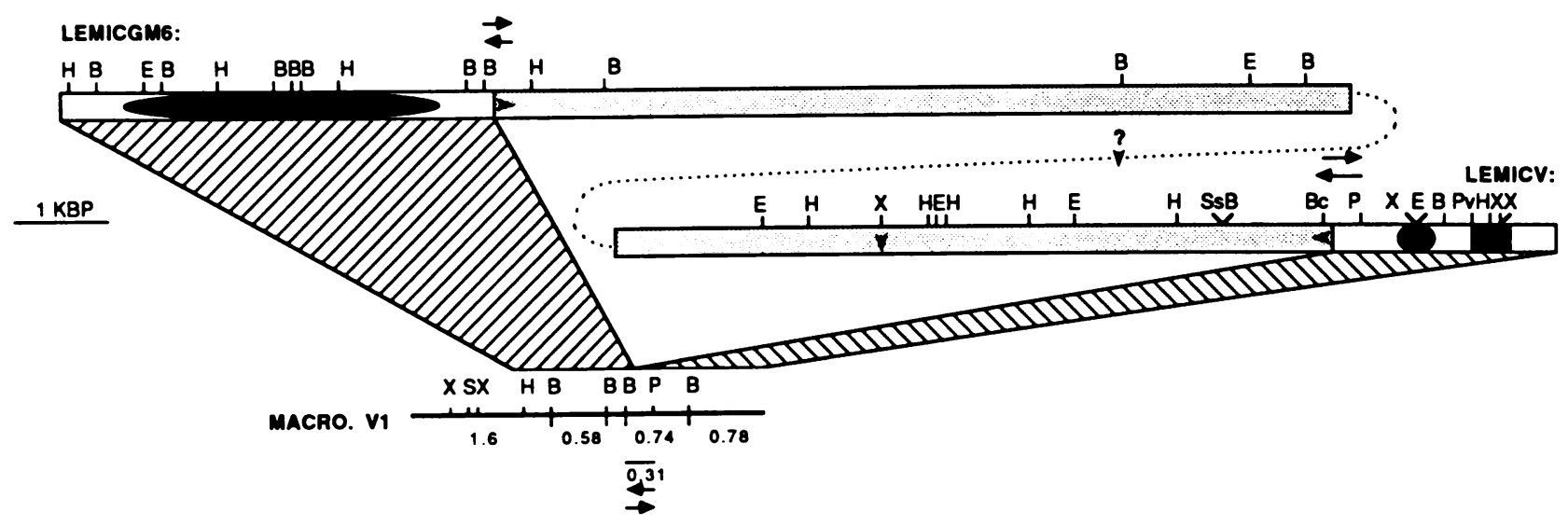

FIG. 7. Restriction map of micronuclear clone LEMICGM6. H. HindIII: E, EcoRI: and B, BglII (not all Bg/II sites were mapped in the right half of the clone). Also shown are the restriction map of macronuclear V1 and a portion of the restriction map of LEMICV, including the region that generates a portion of macronuclear V1. The putative micronuclear orientation of clones LEMICGM6 and LEMICV (.....), regions of the micronuclear clones that form parts of macronuclear V1 ( $)$. IESs in the micronuclear clones where they have been sequenced $(\square)$ and where their precise position is unknown $(O)$, and repetitive DNA sequences $(\square)$ are indicated. The region of each clone sequenced is indicated by arrows, and positions of Tecl element-associated inverted repeats are indicated by arrowheads on the micronuclear restriction maps (arrowheads are pointed downward where the orientation of the inverted repeat is unknown). Sizes of selected restriction fragments referred to in the text are indicated in kilobase pairs.

ently conserved internal restriction sites that place the minimum size of the Tec1 element at $4.7 \mathrm{kbp}$.

The copy number of the repetitive element was determined by hybridizing restriction fragments of LEMICD to restriction digests of whole-cell DNA as well as to restriction digests of known amounts of the LEMICD clone which served as copy-number standards. Figure 6C shows the hybridization of the 1.1-kbp HindIII fragment of LEMICD (fragment C) to HindIII- and EcoRI-digested whole-cell DNA. Densitometric scanning of the major discrete bands on the autoradiogram indicated that there were $1.5 \times 10^{4}$ Tecl elements per micronuclear genome. Similar values were obtained when other LEMICD restriction fragments were used as probes (Table 1). On the basis of a micronuclear DNA content of $1.7 \times 10^{9}$ bp (32) and $1.5 \times 10^{4}$ elements $4.7 \mathrm{kbp}$ in size, we estimate that the element constitutes at least $4.1 \%$ of the micronuclear genome. These values should be considered a minimum estimate of the number of copies of the element in the micronuclear genome for the following reasons: (i) this analysis considers only the major hybridizing bands and not the background smear of hybridization, (ii) the calculation of copy number relies on a minimum estimate for the size of the Tecl element, and (iii) more intense hybridization signals were obtained when the stringency of the hybridization washes was reduced (data not shown).

The Tecl elements interrupting macronuclear precursors resemble transposons. To investigate the interruption in the micronuclear copy of the V1 gene more thoroughly, we sought to isolate a micronuclear clone containing the parts of the V1 precursor not contained in micronuclear clone LEMICV. Therefore, the 2.0-kbp XbaI-PstI fragment of pMACV1 was used to screen the micronuclear recombinant library, which resulted in the isolation of micronuclear clone LEMICGM6 (Fig. 7). When LEMICGM6 was used to probe a Southern blot containing EcoRI and BglII-digested pMACV1 DNA, it hybridized to the V1 restriction fragments that were not detected by LEMICV (Fig. 5B). Additional hybridization experiments were performed to localize the regions of LEMICGM6 sharing homology to V1 and are summarized in Fig. 7. The left end of LEMICGM6 shared homology with V1 extending rightward from the single $H$ indIII site of V1 and into the 0.74 -kbp $B$ glII fragment that is derived in part from LEMICV. Sequences to the left of the HindIII site in macronuclear $\mathrm{V} 1$ were not found in LEMICGM6 but presumably are immediately adjacent to the segment of micronuclear DNA contained in this clone. The region of LEMICGM6 sharing homology with V1 was found to be interrupted by $3.4 \mathrm{kbp}$ of nonrepetitive DNA, which represents one or more IESs (Fig. 7).

The rightmost $8.8 \mathrm{kbp}$ of the micronuclear insert in LEMICGM6 were found to be composed of repetitive DNA (Fig. 7). We suggest that this repetitive region is an extension of the interruption encountered in LEMICV, but this has not been proven since the inserts of LEMICGM6 and LEMICV do not overlap. Repeated attempts to isolate a clone that includes the entire interruption in the V1 precursor were unsuccessful. It is likely that the size of the interruption is larger than the maximum insert size of the cloning vector used to construct the micronuclear library. For purposes of discussion, it will be assumed that the micronuclear sequences represented by the insert of LEMICGM6 are located to the left of the LEMICV insert on the same micronuclear chromosome.

The insertion point of the Tecl element was localized to regions of the two micronuclear clones that give rise to the 0.31-kbp BglII-PstI fragment of the macronuclear V1 DNA molecule (Fig. 7). To examine the ends of the repetitive element, the sequence of the $0.31-\mathrm{kbp} B g$ III-Pst I fragment of pMACV1 was determined, as well as the regions of micronuclear clones LEMICGM6 and LEMICV that contained the ends of the element (Fig. 7 and 8A). The exact length of the macronuclear DNA fragment was found to be $312 \mathrm{bp}$. The first $270 \mathrm{bp}$ of sequence to the left of the single Pst I in LEMICV (Fig. 7) matched the macronuclear sequence (Fig. 8A). Thereafter, the homology to macronuclear V1 ended, which defined the start of the Tecl element (Fig. 8a). The remaining $42 \mathrm{bp}$ required to generate the 312-bp Bg/II-PstI macronuclear fragment were found in the region of LEMICGM6 sequenced, with the homology again breaking down immediately after that sequence. Comparison of the sequences at the ends of the interruption indicated that a 
A.

30

60

90

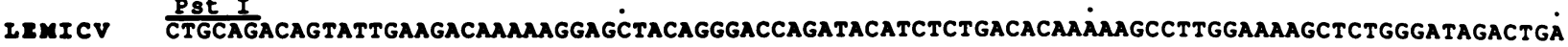

IEMICV AATTTTTGCTTTCAAGTGGTATGAGATTAAAGACATTtTCATAAAAACCAAATGTACCGGTTTCTTTAAAGATtTCCATATCACCTCAA

IEMICV GGATGACTGAAGAAGACATTAGTAATTATGTTGATCCTTTTGCTGTTCTTTCTCAACATGATGGATATAGCTCCGAATTTGCTTCGGAGC

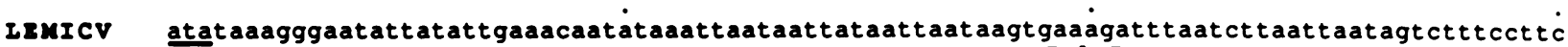

IruICV taccettctagtctcacttaccetccacataagcaagcettaattegcatccttatgatcatcactttgtttgtcttttcagacctt $\dot{\text { Bel }}$

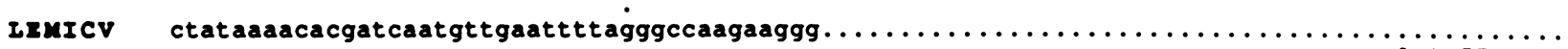

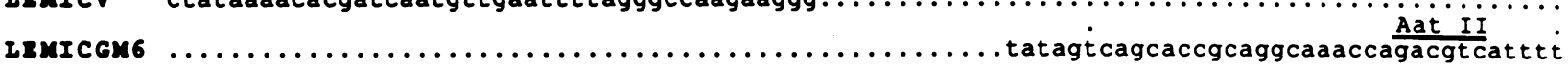

IINICGM 6 agaggctattggcataattcattagaatttggtaatatttgggatagtaaaggtctgaaagacaaacaagtgatgaccataaggat

IIMICGX6 gegaaattatgtcttgcttatgtggagggttaatgagactagaagggtagaaggaagactattaattaagattaaatctt $t c a c t t a t \dot{t}$

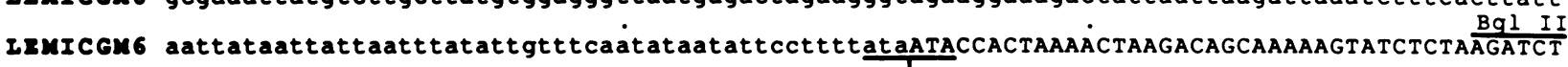

B.

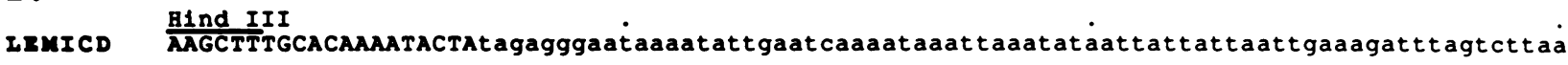

IIXICD ttaattgttattctattacctctctagcctccattagttcccacataggctaactccattccttatcatcatctctctgtattccgt

IXMICD ttaaaccttactatcttcaaatttcccaaactctaatgaattatgctaaatagcctcaaatagctctctgg

c.

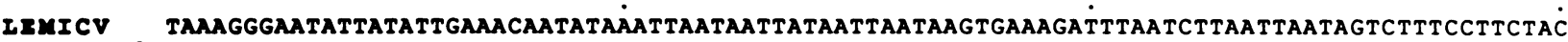
IXMICGM 6 -AAAaGGATATTATATTGAAACAATATAAATTAATAATTATAATTAATAAGTGAAAGATTTAATCTTAATTAATAGTCTTTCCTTCTAC

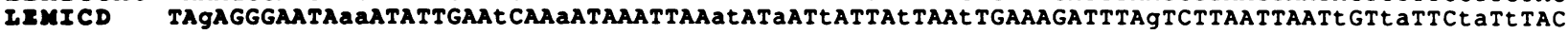

IEMICV CCTTCTAGTCTCACTTA-CCCTCCACATAAGCAAGCCTTAATTTCGCATCCTTATGATCATCACTTTGTTTGTC-TTTTCAGACCTTTAC IINICGM 6 CCTTCTAGTCTCA-TTAaCCCTCCACATAAGCAAGaCaTAATTTCGCATCCTTATGgTCATCACTTTGTTTGTC-TTTTCAGACCTTTAC IEMICD CtCTCTAGCCTCCaTTA-gttCCCACATAgGCtAaCTCCAT------TCCTTATCATCATCtCTCTGTaTtCCgTTTTAAaCCCTTAC

\section{IEMICV TATKIMD OF IUVIRTID RIPIAT}

IIMICGM 6 TATCC-CAAAATATtaCCAAAT TCTAATGAATTATGCCAAATAGCCTCLAAAatGacgTCTGG

IIMICD TATCETCAAAATETCCCCAAACTCTAATGAATTATGCTAAATAGCCTCaAAA T GCt CTCTGG

FIG. 8. Terminal sequences of Tec1 elements in LEMICV, LEMICGM6, and LEMICD. (A) The sequence of LEMICV to the left of the PstI site (Fig. 7) is shown with a portion of the sequence of LEMICGM6 beginning within the Tecl element and extending to a $B g / I I$ site (Fig. 7). Bases which form the 312-bp PstI-Bg/II fragment of macronuclear V1 are shown in uppercase letters, while the terminal bases of the Tec1 element are shown in lowercase letters. The 3-bp direct repeats bracketing the Tecl element are underlined. The sequence of the 312-bp PstI-BglII fragment of pMACV1 is not explicitly shown but it was in complete agreement with the relevant regions of the micronuclear sequences. (B) Sequence of LEMICD beginning at the HindIII site adjacent to the Tecl element (Fig. 1A). The first 20 bp (uppercase letters) generate a portion of the $0.23-\mathrm{kbp}$ HindIII fragment of macronuclear D7, while the bases in lowercase letters constitute the end of the Tec1 element. (C) The sequences of the inverted repeats at the Tec1 element ends in micronuclear clones LEMICV, LEMICGM6, and LEMICD are aligned to display their similarity. Bases in LEMICGM6 and LEMICD which differ from the LEMICV sequence are shown in lowercase letters. Dashes indicate gaps which were introduced into the sequences to obtain maximum alignment. Sequences beyond the inverted repeats are shown for LEMICGM6 and LEMICD to illustrate their similarity (base differences between the two clones are shown in lowercase letters in this region).

3-bp direct repeat $\left(5^{\prime}\right.$-ATA-3') defines the ends of the Tec1 element. For LEMICGM6, there are actually two adjacent 3-bp direct repeats, the first being the last $3 \mathrm{bp}$ of the Tec1 sequences removed during the formation of macronuclear $\mathrm{V} 1$ and the second being the first $3 \mathrm{bp}$ that form macronuclear V1 (Fig. 8A). The overall arrangement is similar to that of IESs, in which one copy of the direct repeat is maintained in the mature macronuclear DNA molecule, although in this instance the double direct repeat in LEMICGM6 complicates the situation. In addition, the $180 \mathrm{bp}$ following the direct repeats in LEMICGM6 and the $181 \mathrm{bp}$ following the direct repeat in LEMICV were found to form an inverted repeat. The two inverted repeats were $96 \%$ identical, differing only by a small number of single-base changes or 1-bp insertions or deletions (Fig. 8C). Thus, the terminal structure of the repetitive element interrupting the $\mathrm{V} 1$ precursor is very similar to that of transposable elements, which frequently have inverted repeats at their ends and create short sequence duplications at their insertion sites $(14,29)$.

In a similar manner, the terminal sequence of one end of the Tec1 element in the precursor of macronuclear D7 was determined (Fig. 1A). In this instance the insertion was localized to a region of LEMICD that gives rise to a part of the 0.23-kbp HindIII fragment of macronuclear D7 (Fig. 1A). Sequencing of the 0.23 -kbp HindIII fragment of LEMACD7 and the corresponding region of LEMICD revealed that LEMICD gives rise to the first $20 \mathrm{bp}$ of the macronuclear DNA fragment, after which the Tec1 element is encountered (Fig. 8B). Since the other end of the D7-Tec1 element has not been cloned, it was not possible to determine whether a direct repeat was present at the end of the element. However, the first 177 bp of the D7-Tec1 are very similar to the inverted repeats of the V1-Tec1 element $(73 \%$ identical to the inverted repeat in LEMICV [Fig. 8C]). Beyond the inverted repeat, the sequence of the D7-Tec1 was very similar to the sequence following the inverted repeat in LEMICGM6 (Fig. 8C). The similarity in sequence beyond the inverted repeat suggests that the Tec1 elements in the V1 and D7 precursors, as shown in the current restriction maps (Fig. 1 and 7), are inserted in opposite orientations.

The conclusion that the Tecl elements are in opposite orientations seemed at odds with the previous cross-hybrid- 


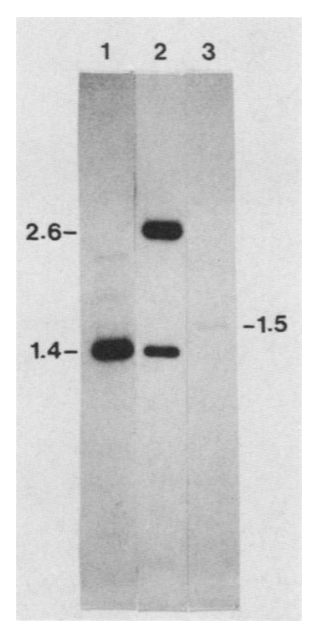

FIG. 9. Hybridization of the $0.28-\mathrm{kbp}$ BglII-Aat II fragment of LEMICGM6, which contains an inverted repeat of the Tec1 element, to a Southern blot containing Bglll-digested LEMICGM6 (lane 1). HindIII- and EcoRI-digested LEMICV (lane 2), and HindIII-digested LEMICD (lane 3). Sizes of hybridizing fragments are indicated in kilobase pairs. The blot was washed in $1 \times$ SSC$0.5 \% \mathrm{SDS}$ at $65^{\circ} \mathrm{C}$. Note that the two light bands seen in lane 1 represent hybridization to fragments generated by partial digestion.

ization experiments between the repetitive regions of LEMICD and LEMICV (Fig. 6A), which indicated that the cross-hybridizing regions were in the same orientation. A possible explanation for this discrepancy arose from further hybridization studies using an inverted-repeat probe. A 0.28-kbp BglII-AatII fragment containing the entire inverted repeat was isolated from LEMICGM6 (Fig. 8A) and used to probe a Southern blot containing the three micronuclear clones (Fig. 9). For LEMICGM6 and LEMICD, only single fragments were detected, corresponding to the positions of the inverted repeats as determined by the sequencing analyses. In a HindIII-EcoRI restriction digest of LEMICV. however, two bands were observed (Fig. 9): a 2.6-kbp HindIII-EcoRI fragment (fragment H, Fig. 1B), which corresponds to the location of the inverted repeat found in the sequence analysis, and a 1.4-kbp HindIII fragment (fragment C, Fig. 1 B). This second fragment defines the end of the LEMICV region that cross-hybridized with LEMICD (Fig. 6A) and is also the fragment that cross-hybridized with fragment $\mathrm{H}$ of LEMICV, which is the fragment that contains the sequenced inverted repeat. A straightforward explanation of these results is that fragment $\mathrm{C}$ contains an additional inverted repeat that defines the start of a second Tec1 element inserted into the outer Tec1 element (Fig. 7). The internal Tecl element would be inversely oriented relative to the external element on the basis of its homology to LEMICD. The left inverted repeat of the proposed internal element has not been detected. It may reside in the uncloned micronuclear DNA separating LEMICGM6 and LEMICV. Alternatively, the left internal inverted repeat could reside in LEMICGM6 but could have diverged sufficiently so that it is no longer detected by the short inverted-repeat probe.

Additional hybridizations of the inverted repeat probe to total cellular DNA were performed to determine whether the inverted repeat was generally associated with the termini of Tec1 elements. Quantitative hybridization experiments with undigested total cellular DNA (performed under reduced stringency and using LEMICGM6 as a copy-number standard) indicated that the repeat was present in $6.4 \times 10^{4}$ copies per cell (Table 1). This number is consistent with copies of the inverted repeat residing at both ends of other micronuclear Tecl elements. Hybridization of the inverted repeat probe to total cellular DNA digested with EcoRI. $B g l \mathrm{II}, X b a \mathrm{I}$, or HincII produced only a smear of hybridization with no discrete fragments detectable (Table 1: data not shown). This is consistent with a terminal location for the inverted repeat on Tecl elements, as the positions of restriction sites in micronuclear DNA flanking the Tecl elements are expected to be variable. However, when the inverted repeat probe was hybridized to HindIII-digested total cellular DNA, two discrete fragments of 1.9 and $1.4 \mathrm{kbp}$ were observed above the background smear (Table 1). This result suggests that many Tecl elements contain a HindIII site within their inverted repeats and that the 1.9- and 1.4-kbp HindIII fragments represent the two immediately subterminal HindIII fragments of the conserved Tecl elements in the genome.

The V1 Tecl element is reproducibly excised during macronuclear development. The ability to isolate sexual offspring of $E$. crassus made it possible to determine whether the Tec1 element was reproducibly removed from the V1 precursor during macronuclear development. To do this, it was first necessary to determine whether all micronuclear copies of $\mathrm{V} 1$ in two cell lines of opposite mating type contained a Tec1 element. This was examined by hybridizing the 700-bp $B g$ lII-PstI fragment of LEMICV (Fig. 1B) to restriction digests of $E$. crassus micronuclear DNA isolated from strains $\mathrm{G} 1$ and $\mathrm{NcC10}$ (Fig. 10A and $\mathrm{B}$ ). The restriction digests and probe were chosen so as to detect fragments which both contained homology to V1 and overlapped the right end of the Tec1 insertion. Fragments of 2.2, 3.4, and 5.7 kbp were detected in $B g / I I$, HindIII, and $X b a I$ digests, respectively, of micronuclear DNA isolated from both strains of E. crassus. The sizes of these fragments were all in agreement with the map of LEMICV (Fig. 1B), indicating that both strains have a micronuclear copy of $\mathrm{V} 1$ containing a Tec1 element. However, an additional band was also observed in each micronuclear digest (Fig. 10A and B). The sizes of these second bands corresponded to the fragments that would be derived from the macronuclear copy of $V 1$ if it was digested with each enzyme. Thus, these additional fragments likely represent a small amount of macronuclear DNA contamination in the micronuclear DNA preparations rather than a second micronuclear form of $\mathrm{V} 1$ that lacks a Tecl element. This conclusion is supported by the observation that the additional bands varied in intensity in independent preparations of micronuclear DNA (e.g. compare Fig. $10 \mathrm{~A}$ and $\mathrm{B}$; data not shown). Overall, the results indicated that all micronuclear copies of V1 are interrupted by a Tec1 element.

To determine whether the Tec 1 element was reproducibly removed from the V1 precursor during macronuclear development. strains $\mathrm{Gl}$ and $\mathrm{NcC10}$ were mated and four independent cell lines were established from the sexual offspring (strains CC102, CC103, CC104, and CC106). A Southern blot containing BglII-digested whole-cell DNA from each of these new cell lines was then constructed and probed with the 2.5-kbp HindIII-EcoRI (right terminus) fragment of clone pMACV1. For each cell line, four fragments were detected in the hybridization analysis (Fig. 10C) which all had sizes predicted from the restriction map of macronuclear V1 (Fig. 7). These included a $0.74-\mathrm{kbp} B g$ lII fragment, which corresponds to the site of the Tec1 insertion in the micronuclear copy of V1 and whose size would be altered if the Tec1 element were not removed. The results thus indicate that the 


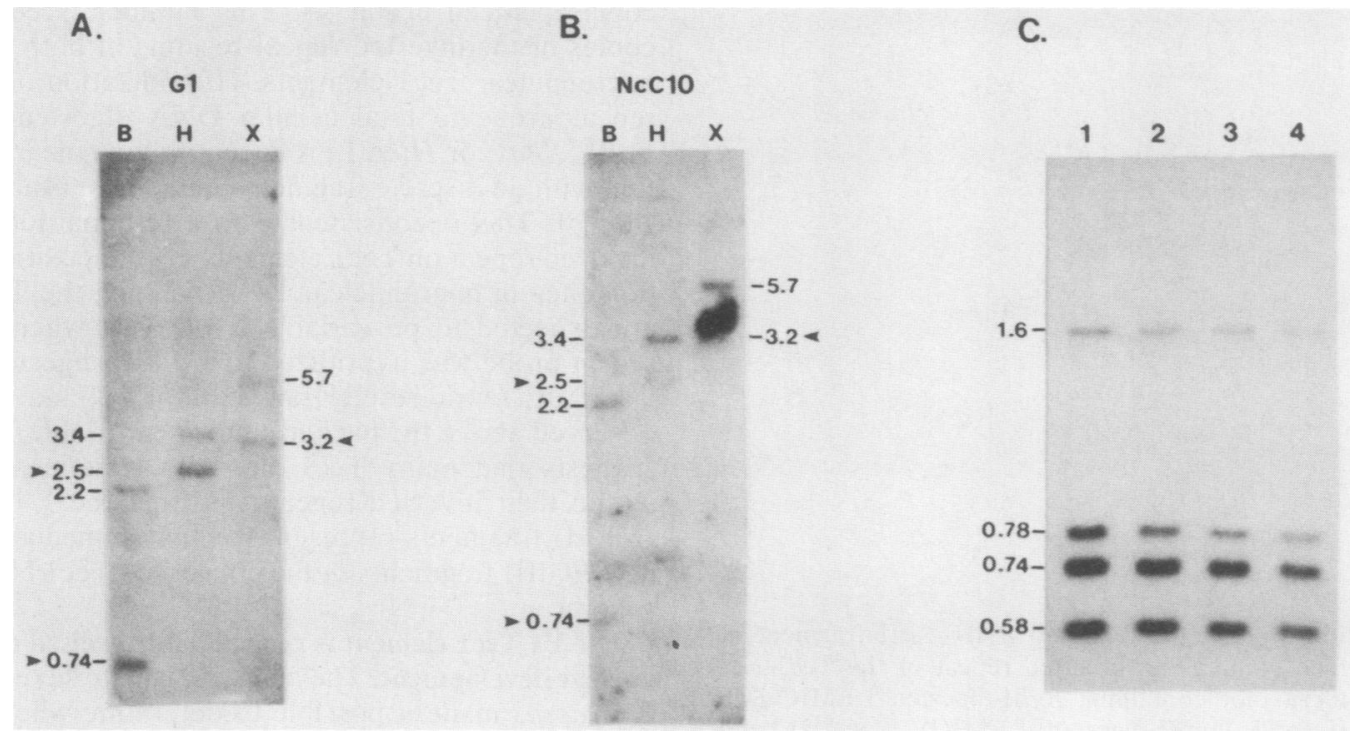

FIG. 10. (A) Hybridization of the 700-bp BglII-PstI fragment of LEMICV to a Southern blot containing micronuclear DNA of $E$. crassus G1 digested with BglII (lane B), HindIII (lane H), and XbaI (lane X). (B) Hybridization of the 700-bp BglII-PstI fragment of LEMICV to a Southern blot containing micronuclear DNA of E. crassus NcC10 digested with BglII (lane B), HindIII (lane H), and XbaI (lane X). (C) Hybridization of the $2.5-\mathrm{kbp}$ HindIII-EcoRI (right terminus) fragment of pMACV1 to a Southern blot containing Bg/II digested whole-cell DNA of E. crassus CC102 (lane 1), CC103 (lane 2), CC104 (lane 3), and CC106 (lane 4). In each case, sizes of hybridizing fragments are indicated in kilobase pairs. For A and B, hybridizing fragments resulting from macronuclear contamination of the micronuclear DNA preparations are indicated by arrowheads.

Tec1 element is removed from the micronuclear copy of V1 during independent episodes of macronuclear development. As there were no detectable size differences in the $0.74-\mathrm{kbp}$ $B g l$ II fragments, the removal of the Tecl element appears to have been similar in each of the cell lines. However, the hybridization method is not sensitive enough to conclude that removal occurred precisely in each of the cell lines.

\section{DISCUSSION}

Micronuclear genome organization in $E$. crassus. The organization of two cloned segments of $E$. crassus micronuclear DNA that contain precursors of macronuclear DNA molecules has been examined. The overall results indicate that the micronuclear precursors of macronuclear DNA molecules are organized in a manner very similar to those of previously studied hypotrich species (see Introduction), particularly the well-studied O. nova. Each of the two clones possessed more than one macronuclear precursor; LEMICD gives rise to both macronuclear D8 and a part of D7, while LEMICV generates at a minimum all or parts of macronuclear V1, V2, and V3. Thus, the precursors of macronuclear DNA molecules appear to be clustered in the micronuclear chromosomes rather than separated by large segments of DNA that are eliminated during macronuclear development. Sequence analysis indicated that the precursors are indeed closely spaced. D7 and D8 are separated by only $36 \mathrm{bp}$, while the precursors of V2 and V3 are separated by $400 \mathrm{bp}$. The precursors of V1 and V2 were found to overlap by $6 \mathrm{bp}$. Evidence for overlapping precursors has previously been obtained for $O$. nova (30), but the situation was complicated by the presence of multiple variant forms of the macronuclear gene precursors analyzed. Since our data are consistent with only single forms of V1 and V2, the sequence data provide strong evidence that macronuclear gene precursors can overlap. Models for the resolution of overlapping macronuclear precursors are discussed elsewhere (7).
The sequence analysis also indicated that macronuclear gene precursors completely lack telomeric repeat sequences, which is consistent with results obtained for other hypotrich species $(11,31,39)$ and $T$. thermophila (51) and reinforces the conclusion that the telomeric repeats are added to macronuclear DNA molecules during development. The current analysis also provides the sequences in the vicinity of a number of chromosome fragmentation sites and the opportunity to search for conserved sequences that might be involved in directing chromosome fragmentation. This type of analysis has been done and is discussed in conjunction with data on the fidelity of the chromosome fragmentation process in E. crassus (7).

An additional feature of the precursors of macronuclear DNA molecules is the frequent occurrence of IESs. The precursors of the five cloned macronuclear DNA molecules contain a minimum of eight unique or low-copy-number IESs. Although data concerning the number of macronuclear DNA molecules in $E$. crassus are unavailable, all hypotrichs examined to date have 10,000 to 40,000 macronuclear DNA molecules (32). Assuming that E. crassus has a similar number, extrapolation of our results suggests that tens of thousands of IESs exist in the micronuclear genome and are removed during development. The sizes of the sequenced IESs ranged from 31 to $374 \mathrm{bp}$, which is similar to the sizes of IESs in Oxytricha species $(23,40)$. On the basis of hybridization analyses, even larger conventional IESs may exist (Fig. 1). The three sequenced IESs are bounded by direct repeats of 2 to $4 \mathrm{bp}$, and two of them contain short, imperfect inverted repeats near their termini, which are also features of Oxytricha IESs. Our results also indicate that the macronuclear precursor of D8 lacks IESs. This is the first instance in any hypotrichous ciliate where a macronuclear precursor has been found to be free of IESs. Interestingly, there is an approximately sixfold overamplification of D8 relative to most other macronuclear DNA molecules during 
macronuclear development (S. E. Baird and L. A. Klobutcher, unpublished results). It is not clear whether the absence of IESs in the D8 precursor is related to its developmental overamplification.

Preliminary evidence also indicates that alternative processing occurs in $E$. crassus, as has been observed in other hypotrichs $(23,30)$. Both macronuclear V3 and V4 showed strong hybridization to the same region of LEMICV. On the basis of hybridization analyses, both the alternative use of chromosome fragmentation sites and alternative internal rearrangements would be required to generate these two macronuclear DNA molecules. This suggests that internal rearrangements may influence the position of chromosome fragmentation. Forney and Blackburn (15) have observed a similar phenomena in $P$. tetraurelia. V3 and V4 provide a potential opportunity to further investigate the interrelationship of the two processes.

The results from our two micronuclear clones are also consistent with those of a general survey of $E$. crassus micronuclear genome organization recently reported by Jahn et al. (25). In this study, the general organization of 24 micronuclear clones was examined by hybridization analyses with total cellular DNA. The cloned segments of micronuclear DNA fell into three classes: (i) one-fourth of the clones contained only unique micronuclear DNA sequences eliminated during development; (ii) approximately one-half of the clones contained a large repetitive DNA element; and (iii) one-third of the clones contained both the large repetitive element and homology to multiple macronuclear DNA molecules. The two clones analyzed in this study would fall into the third category, as they have both multiple macronuclear precursors and repetitive DNA. Although we have analyzed only two cloned segments of micronuclear DNA in detail, the frequent observation of a similar arrangement by Jahn et al. (25) indicates that the features displayed by LEMICD and LEMICV are generally representative of regions of the micronuclear genome containing macronuclear precursors.

Overall, these two E. crassus micronuclear clones display all the organizational and rearrangement features that have been found previously in other hypotrich species. It will thus be possible to use this organism to generate large numbers of cells synchronously undergoing macronuclear development (42) to further study the timing and mechanisms of DNA rearrangement processes in hypotrichs.

Developmental excision of a transposonlike element from a macronuclear precursor. The most unexpected finding during the analysis of LEMICD and LEMICV was the observation of related blocks of repetitive DNA interrupting the precursors of macronuclear D7 and V1. Analysis of these repetitive regions indicated that they are degenerate members of a family of large repetitive elements present in at least 10,000 copies in the micronuclear genome. In the case of $\mathrm{V} 1$, the interruption is actually a composite of two, and possibly more, repetitive elements. All micronuclear copies of $\mathrm{V} 1$ contain the repetitive element, and it is reproducibly removed from the V1 precursor during independent episodes of macronuclear development. Through sequence analysis of a macronuclear clone and a micronuclear clone, the V1 Tec1 element was found to be bounded by a direct repeat of $3 \mathrm{bp}$. In addition, the ends of the V1 element and one end of the D7 element possess an inverted repeat of approximately $180 \mathrm{bp}$. This terminal organization is very similar to that of many transposable elements $(14,29)$, some of which have been shown to undergo excision (e.g., 12, 38) which resembles the Tec1 removal process. Although movement of the element to new sites in the genome has not been demonstrated, the terminal structure of the element and its developmental removal both suggest that the $E$. crassus repetitive DNA element may be a transposon.

The repetitive elements in LEMICD and LEMICV are, in fact, the same repetitive DNA elements identified by Jahn et al. (25). This was first suggested by similarities in the size, copy number, and consensus restriction sites of the element characterized above to those previously reported by Jahn et al. (25). To more clearly demonstrate the identity of the elements, these workers have hybridized probes derived from their repetitive DNA elements to our two clones and observed strong hybridization (L. A. Nilles and C. L. Jahn, personal communication). We have jointly agreed to refer to the repetitive blocks of DNA as Tec1 (for Transposonlike element, $E$. crassus) elements.

In their study, Jahn et al. (25) used hybridization analyses to demonstrate that Tecl elements were frequently associated with clusters of macronuclear precursors in a series of randomly selected micronuclear clones. More detailed hybridization analysis of one micronuclear clone suggested that the Tec1 element interrupted the precursor of a macronuclear DNA molecule in a manner similar to that which we have observed. In combination with our data, these results suggest that Tecl elements may frequently interrupt macronuclear gene precursors.

Repetitive elements have been observed in other hypotrich species. In $O$. nova, a $25-\mathrm{kbp}$ repetitive element is preferentially associated with clusters of macronuclear precursors $(9,26)$. The preferential association of this element with macronuclear precursors suggests that it also may interrupt them, but detailed studies of micronuclear clones harboring the element have yet to be performed. A situation that is more clearly relevant to what we have found in $E$. crassus exists in $O$. fallax. Herrick et al. (22) have defined a family of transposonlike elements (TBE [telomere-bearing elements]) in the micronuclear genome of $O$. fallax that are eliminated during macronuclear development. Two such TBE1 elements were found in sequences that hybridized to a family of macronuclear DNA molecules generated by alternative processing. It was initially unclear whether the micronuclear sequences containing the elements actually generated the homologous macronuclear DNA molecules because related micronuclear loci lacking the TBE1 elements were found that do give rise to members of the family of macronuclear DNA molecules. More recent data from the laboratory of G. Herrick (D. Hunter, S. Cartinhour, and G. Herrick, personal communication) suggest that the TBE1bearing locus is also active in generating macronuclear DNA molecules, implying that the TBE1 elements can also be developmentally removed from macronuclear gene precursors.

Developmental removal of transposable elements is not limited to the hypotrichous ciliates. A family of transposons has been identified in the holotrichous ciliate $T$. thermophila that is largely eliminated during development of the macronucleus (10). In addition, at least some copies of a retroviruslike element in the nematode Ascaris lumbricoides are eliminated from somatic cells but not from germ cells (1). In these cases it is not clear whether the element excises independently or is simply eliminated because it is embedded in other sequences destined to undergo developmental elimination.

We and others have previously pointed out the similarity of IESs and transposable elements on the basis of terminal structure and suggested that the two might be related $(22$, 
40). That is, IESs might be degenerate transposable elements that retain cis-acting sequences necessary for excision, but intact elements present elsewhere in the genome would be required to encode trans-acting excision factors. In $O$. nova, no evidence for this scenario was obtained, as hybridization studies indicated that the IESs are unique sequences in the micronuclear genome (40). The observation that $E$. crassus contains conventional IESs (unique sequences) and Tec1 elements, which are both removed from macronuclear precursors, again raises the possibility that the two are related. Although no significant sequence similarity is consistently observed between internal regions of the IESs and the sequenced regions of the V1-Tec1 element, the direct repeats that bound the IESs (5'-ATAT-3', 5'-TA-3', and 5'ATA-3') and the V1-Tec1 (5'-ATA-3') are similar. All have direct repeats that consist of two to four AT base pairs, and the dinucleotide 5'-TA-3' is shared by all the direct repeats. If the direct repeats arise by target site duplication during insertion, as is the case with conventional transposable elements, it is possible that both the IESs and Tec1s insert specifically at a 5'-TA-3' genomic site, creating a 2-bp duplication. The fact that the V1 Tec1 and some of the IESs have longer direct repeats is not inconsistent with this, as the length of the repeats we observed was, in effect, a maximum length. In some cases, one expects nucleotides preceding or following the functional direct repeats to be identical by chance, giving the appearance of a longer direct repeat. Interestingly, the Tc1 transposable element of Caenorhabditis elegans appears to integrate exclusively at $5^{\prime}$-TA-3' genomic sites (21).

The removal of Tec1 elements and IESs from macronuclear precursors is also similar to developmental DNA breakage and joining processes that are required for gene assembly and expression. These include rearrangements involving a gene encoding a sporulation-specific sigma factor in Bacillus subtilis (48), nitrogen fixation genes in Anabaena spp. (18), and immunoglobulin and T-cell receptor genes in vertebrates (2). We expect that some Tec1 elements will be found to interrupt the coding regions of macronuclear gene precursors, so that Tec1 removal may represent an analogous means of activating genes during macronuclear development. Alternatively, it has been noted that the ciliate micronucleus is an ideal haven for selfish DNA $(25,40)$. Because the micronuclear genome is transcriptionally inactive, insertion of sequences such as IESs and Tec1s into the precursors of macronuclear DNA molecules would have no immediate effect on the cell. If such sequences possess the ability to be removed during macronuclear development, they would also have little long-term effect on the organism and would be expected to proliferate in the micronucleus.

It is possible that the Tec1 elements are at least partially responsible for their own removal. Some transposable elements are known to encode factors involved in their excision (41). Similarly, in Anabaena spp., a gene has been located within eliminated DNA that is required for the DNA breakage and joining event which forms an intact nitrogen fixation gene (18). If Tec1 elements are indeed transposons that encode excision functions, further study of these elements may provide insight not only into how they themselves are developmentally removed but also into how IESs are removed. It will be particularly important to determine whether Tec1 elements are transcriptionally activated during macronuclear development and whether they exist as free forms. Again, the ability to produce synchronous cultures of E. crassus undergoing macronuclear development will be extremely useful in this regard.

\section{ACKNOWLEDGMENTS}

We thank G. Herrick and A. Cowan for their suggestions on the manuscript. and we gratefully acknowledge the technical assistance of J. Murphy and N. Hyde-DeRuyscher.

This work was supported by Public Health Service grant GM33277 from the National Institutes of Health and by a grant from the University of Connecticut Research Foundation to L.A.K.

\section{LITERATURE CITED}

1. Aeby, P., A. Spicher, Y. de Chastonay, F. Muller, and H. Tobler. 1986. Structure and genomic organization of proretrovirus-like elements partially eliminated from the somatic genome of $A s$ caris lumbricoides. EMBO J. 5:3353-3360.

2. Alt, F. W., T. K. Blackwell, and G. D. Yancopoulos. 1987. Development of the primary antibody repertoire. Science 238: 1079-1087.

3. Altschuler, M. I., and M.-C. Yao. 1985. Macronuclear DNA of Tetrahymena thermophila exists as defined subchromosomalsized molecules. Nucleic Acids Res. 13:5817-5831.

4. Ammermann, D. 1987. Germ line specific DNA and chromosomes of the ciliate Stylonychia lemnae. Chromosome 95:37-43.

5. Ammermann, D., and A. Muenz. 1982. DNA and protein content of different hypotrich ciliates. Eur. J. Cell Biol. 27:22-24.

6. Baird, S. E., and L. A. Klobutcher. 1988. Genetic characterization and use of a restriction fragment length variant in the hypotrichous ciliate Euplotes crassus. J. Protozool. 35:459-465.

7. Baird, S. E., and L. A. Klobutcher. 1989. Characterization of chromosome fragmentation in two protozoans and identification of a candidate fragmentation sequence in Euplotes crassus. Genes Dev. 3:585-597.

8. Benton, W. D., and R. W. Davis. 1977. Screening lambda gt10 recombinant clones by hybridization to single plaques in situ. Science 196:180-182

9. Boswell, R. E., C. L. Jahn, A. F. Greslin, and D. M. Prescott. 1983. Organization of gene and non-gene sequences in micronuclear DNA of Oxytricha nova. Nucleic Acids Res. 11:36513663.

10. Cherry, J. M., and E. H. Blackburn. 1985. The internally located telomeric sequences in the germ-line chromosomes of Tetrahymena are at the ends of transposon-like elements. Cell 43:747-758.

11. Dawson, D., and G. Herrick. 1984. Rare internal $C_{4} A_{4}$ repeats in the micronuclear genome of Oxytricha fallax. Mol. Cell. Biol. 4:2661-2667.

12. Emmons, S. W., and L. Yesner. 1984. High-frequency excision of transposable element $\mathrm{Tcl}$ in the nematode Caenorhabditis elegans is limited to somatic cells. Cell 36:599-605.

13. Feinberg, A. P., and B. Vogelstein. 1983. A technique for radiolabeling DNA restriction endonuclease fragments to high specific activity. Anal. Biochem. 132:6-13. (Addendum, 137: 266-267).

14. Finnegan, D. J. 1985. Transposable elements in eukaryotes. Int. Rev. Cytol. 93:281-326.

15. Forney, J. D., and E. H. Blackburn. 1988. Developmentally controlled telomere addition in wild-type and mutant Paramecia. Mol. Cell. Biol. 8:251-258.

16. Frischauf, A., H. Lehrach, A. Poustka, and N. Murray. 1983. Lambda replacement vectors carrying polylinker sequences. J. Mol. Biol. 170:827-842.

17. Godson, G. N., and D. Vapnek. 1973. A simple method of preparing large amounts of $\phi X 174$ RFI supercoiled DNA. Biochim. Biophys. Acta 299:516-520.

18. Golden, J. W., and D. R. Wiest. 1988. Genome rearrangement and nitrogen fixation in Anabaena blocked by inactivation of $X$ is A gene. Science 242:1421-1423.

19. Heckmann, K. 1964. Experimentelle untersuchungen an Euplotes crassus. I. Paarungssystem, konjugation und determination der paarungstypen. Z. Verebungsl. 95:114-124.

20. Helftenbein, E. 1985. Nucleotide sequence of a macronuclear DNA molecule coding for $\alpha$-tubulin from the ciliate Stylonychia lemnae. Nucleic Acids Res. 13:415-433.

21. Herman, R. K., and J. E. Shaw. 1987. The transposable genetic 
element $\mathrm{Tcl}$ in the nematode Caenorhabditis elegans. Trends Genet. 3:222-225.

22. Herrick, G., S. Cartinhour, D. Dawson, D. Ang, R. Sheets, A. Lee, and K. Williams. 1985. Mobile elements bounded by $C_{4} A_{4}$ telomeric repeats in Oxytricha fallax. Cell 43:759-768.

23. Herrick, G., D. Hunter, K. Williams, and K. Kotter. 1987. Alternative processing during development of a macronuclear chromosome family in Oxytricha fallax. Genes Dev. 1:10471058.

24. Huynh, T., R. A. Young, and R. W. Davis. 1985. Construction and screening cDNA libraries in lambda GT10 and lambda GT11, p. 49-78. In D. M. Glover (ed.), DNA cloning: a practical approach. IRL Press, Ltd., Oxford.

25. Jahn, C. L., L. A. Nilles, and M. F. Krikau. 1988. Organization of the Euplotes crassus micronuclear genome. J. Protozool. 35:590-601.

26. Jahn, C. L., K. E. Prescott, and M. W. Waggener. 1988. Organization of the micronuclear genome of Oxytricha nova. Genetics 120:123-134.

27. Kaine, B. P., and B. B. Spear. 1982. Nucleotide sequence of a macronuclear gene for actin in Oxytricha fallax. Nature (London) 295:430-432.

28. Karrer, K. M. 1986. The nuclear DNAs of holotrichous ciliates, p. 85-110. In J. Gall (ed.), The molecular biology of ciliated protozoa. Academic Press, Inc., New York.

29. Kleckner, N. 1981. Transposable elements in prokaryotes. Annu. Rev. Genet. 15:341-404.

30. Klobutcher, L. A., M. E. Huff, and G. E. Gonye. 1988. Alternative use of chromosome fragmentation sites in the ciliated protozoan Oxytricha nova. Nucleic Acids Res. 16:251-264.

31. Klobutcher, L. A., C. J. Jahn, and D. M. Prescott. 1984. Internal sequences are eliminated from genes during macronuclear development in the ciliated protozoan Oxytricha nova. Cell 36: $1045-1055$

32. Klobutcher, L. A., and D. M. Prescott. 1986. The special case of the hypotrichs, p. 111-154. In J. Gall (ed.), The molecular biology of ciliated protozoa. Academic Press, Inc., New York.

33. Klobutcher, L. A., M. S. Swanton, P. Donini, and D. M. Prescott. 1981. All gene-sized DNA molecules in four species of hypotrichs have the same terminal sequence and an unusual 3' terminus. Proc. Natl. Acad. Sci. USA 78:3015-3019.

34. Klobutcher, L. A., A. M. Vailonis-Walsh, K. Cahill, and R. M. Ribas-Aparicio. 1986. Gene-sized maronuclear DNA molecules are clustered in micronuclear chromosomes of the ciliate $O x$ ytricha nova. Mol. Cell. Biol. 6:3606-3613.

35. Kuhn, S., H.-J. Fritz, and P. Starlinger. 1979. Close vicinity of IS1 integration sites in the leader sequence of the gal operon of E. coli. Mol. Gen. Genet. 167:235-241.

36. Maniatis, T., E. F. Fritsch, and J. Sambrook. 1982. Molecular cloning: a laboratory manual. Cold Spring Harbor Laboratory, Cold Spring Harbor, N.Y.
37. Maxam, A. M., and W. Gilbert. 1980. Sequencing end-labeled DNA with base-specific chemical cleavages. Methods Enzymol. 65:499-560.

38. O'Hare, K., and G. M. Rubin. 1983. Structure of $P$ transposable elements and their sites of insertion and excision in the Drosophila melanogaster genome. Cell 34:25-35.

39. Oka, Y., and T. Honjo. 1983. Common terminal repeats of the macronuclear DNA are absent from the micronuclear DNA in hypotrichous ciliate Stylonychia pustulata. Nucleic Acids Res. 11:4325-4333.

40. Ribas-Aparicio, R. M., J. J. Sparkowski, A. E. Proulx, J. D. Mitchell, and L. A. Klobutcher. 1987. Nucleic acid splicing events occur frequently during macronuclear development in the protozoan Oxytricha nova and involve the elimination of unique DNA. Genes Dev. 1:323-336.

41. Rio, D. C., F. A. Laski, and G. M. Rubin. 1986. Identification and immunochemical analysis of biologically active Drosophila P element transposase. Cell 44:21-32.

42. Roth, M., M. Lin, and D. M. Prescott. 1985. Large scale synchronous mating and the study of macronuclear development in Euplotes crassus. J. Cell Biol. 101:79-84.

43. Roth, M. R., and D. M. Prescott. 1985. DNA intermediates and telomere addition during genome reorganization in Euplotes crassus. Cell $41: 411-417$.

44. Sanger, F., S. Nicklen, and A. Coulson. 1977. DNA sequencing with chain-terminating inhibitors. Proc. Natl. Acad. Sci. USA 74:5463-5467.

45. Sheperd, J. C. W. 1981. Method to determine the reading frame of a protein from the purine/pyrimidine genome sequence and its possible evolutionary justification. Proc. Natl. Acad. Sci. USA 78:1596-1600.

46. Southern, E. M. 1975 . Detection of specific sequences among DNA fragments separated by gel electrophoresis. J. Mol. Biol. 98:503-517.

47. Steinbruck, G. 1986. Molecular reorganization during nuclear differentiation in ciliates, p. 105-174. In W. Hennig (ed.), Results and problems in cell differentiation, vol. 13. Germ line-soma differentiation. Springer-Verlag KG, Berlin.

48. Stragier, P., B. Kunkel, L. Kroos, and R. Losick. 1989. Chromosomal rearrangement generating a composite gene for a developmental transcription factor. Science 243:507-512.

49. Vieira, J., and J. Messing. 1982. The pUC plasmids, an M13mp7-derived system for insertion mutagenesis and sequencing with universal primers. Gene 19:259-268.

50. Yao, M.-C., J. Choi, S. Yokoyama, C. F. Austerberry, and C.-H. Yao. 1984. DNA elimination in Tetrahymena: a developmental process involving extensive breakage and rejoining of DNA at defined sites. Cell 36:433-440.

51. Yao, M.-C., K. Zheng, and C.-H. Yao. 1987. A conserved nucleotide sequence at the sites of developmentally regulated chromosome breakage in Tetrahymena. Cell 48:779-788. 